PREPARED FOR SUBMISSION TO JHEP

\title{
Holographic Charged Rényi Entropies
}

\author{
Alexandre Belin, ${ }^{a, b}$ Ling-Yan Hung, ${ }^{b}$ Alexander Maloney, ${ }^{a, b}$ Shunji Matsuura, ${ }^{a, b}$ \\ Robert C. Myers ${ }^{c}$ and Todd Sierens ${ }^{c, d}$ \\ ${ }^{a}$ Departments of Physics and Mathematics, McGill University, Montréal, Québec, Canada \\ ${ }^{b}$ Department of Physics, Harvard University, Cambridge, MA 02138 USA \\ ${ }^{c}$ Perimeter Institute for Theoretical Physics, Waterloo, Ontario N2L 2Y5, Canada \\ ${ }^{d}$ Department of Physics 8 Astronomy and Guelph-Waterloo Physics Institute, University of \\ Waterloo, Waterloo, Ontario N2L 3G1, Canada \\ E-mail: alexandre.belin@mail.mcgill.ca, lhung@physics.harvard.edu, \\ maloney@physics.mcgill.ca, matsuura@physics.mcgill.ca, \\ rmyers@perimeterinstitute.ca, tsierens@perimeterinstitute.ca
}

\begin{abstract}
We construct a new class of entanglement measures by extending the usual definition of Rényi entropy to include a chemical potential. These charged Rényi entropies measure the degree of entanglement in different charge sectors of the theory and are given by Euclidean path integrals with the insertion of a Wilson line encircling the entangling surface. We compute these entropies for a spherical entangling surface in CFT's with holographic duals, where they are related to entropies of charged black holes with hyperbolic horizons. We also compute charged Rényi entropies in free field theories.
\end{abstract}

ArXiv EPrint: 1310.4180 


\section{Contents}

1 Introduction $\quad 2$

2 Charged Rényi entropies for CFT's $\quad 4$

2.1 Replica Trick 4

2.2 Spherical entangling surfaces 6

2.3 Properties of generalized twist operators 9

2.4 Generalized twist operators in $d=2 \quad 16$

3 Holographic computations $\quad 19$

3.1 Charged black hole solution 20

3.2 Charged Rényi entropies 22

3.3 Twist operators $\quad 26$

3.4 Thermodynamics, stability and phase transitions 27

4 Discussion $\quad 29$

A Free QFT computations $\quad 31$

A.1 Heat kernels on $S^{1} \times H^{d-1}$

A.1.1 $d=2 \quad 33$

A.1.2 $d=4 \quad 34$

A.2 Wavefunctions on $S^{1} \times H^{d-1} \quad 35$

A.2.1 Free scalar field 36

A.2.2 Free Dirac fermion 37

$\begin{array}{lll}\text { A.3 Wavefunctions on } S^{d} & 39\end{array}$

A.3.1 Free scalar field 39

A.3.2 Free Dirac fermion 42

B Holographic computations for $d=2 \quad 44$

B.1 Einstein-Maxwell theory 45

B.2 Einstein-Chern-Simons theory 48

C Holographic minutiae $\quad 49$

C.1 Energy density 49

C.2 Charge density $\quad 50$

C.3 Boundary CFT parameters $\quad 51$ 


\section{Introduction}

Entanglement and Rényi entropies have emerged as diagnostic probes of considerable practical and formal interest in areas ranging from condensed matter physics, e.g., $[1,2]$ to quantum gravity, e.g., [3-5]. In this paper we will consider a generalization of these entropies for systems with a conserved global charge.

Consider a quantum system consisting of two components, $\mathrm{A}$ and $\mathrm{B}$, in a state described by the density matrix $\rho$. We will consider quantum field theories, where A and $\mathrm{B}$ are spatial regions separated by an entangling surface $\Sigma$. We then trace over the degrees of freedom in region B to construct the reduced density matrix $\rho_{\mathrm{A}}=\operatorname{Tr}_{\mathrm{B}} \rho$. The latter contains information about the entanglement between $\mathrm{A}$ and $\mathrm{B}$. The Rényi entropies $[6,7]$

$$
S_{n}=\frac{1}{1-n} \log \operatorname{Tr} \rho_{\mathrm{A}}^{n},
$$

are the moments of this reduced density matrix. The limit $n \rightarrow 1$ gives the entanglement entropy, $S_{\mathrm{EE}}=\lim _{n \rightarrow 1} S_{n}=-\operatorname{Tr}\left[\rho_{\mathrm{A}} \log \rho_{\mathrm{A}}\right]$.

In this paper, we will consider quantum field theories with a conserved (global) charge. In this case we can ask whether the entanglement between A and B depends on how charge is distributed between the two subsystems. This is characterized by the following 'grand canonical' generalization of the Rényi entropy:

$$
S_{n}(\mu)=\frac{1}{1-n} \log \operatorname{Tr}\left[\rho_{\mathrm{A}} \frac{e^{\mu Q_{\mathrm{A}}}}{n_{\mathrm{A}}(\mu)}\right]^{n} .
$$

Here $\mu$ is a chemical potential conjugate to $Q_{\mathrm{A}}$, the charge contained in subsystem A. We have also introduced $n_{\mathrm{A}}(\mu) \equiv \operatorname{Tr}\left[\rho_{\mathrm{A}} e^{\mu Q_{\mathrm{A}}}\right]$ to ensure that the new density matrix (enclosed by the square brackets above) is properly normalized with unit trace. The $\mu$-dependence of these 'charged' Rényi entropies $S_{n}(\mu)$ encodes the dependence of the entanglement on the charge.

We will also be interested in the entropies constructed with an imaginary chemical potential

$$
\tilde{S}_{n}\left(\mu_{\mathrm{E}}\right)=\frac{1}{1-n} \log \operatorname{Tr}\left[\rho_{\mathrm{A}} \frac{e^{i \mu_{\mathrm{E}} Q_{\mathrm{A}}}}{\tilde{n}_{\mathrm{A}}\left(\mu_{\mathrm{E}}\right)}\right]^{n},
$$

where $\mu_{\mathrm{E}}$ is real and $\tilde{n}_{\mathrm{A}}(\mu) \equiv \operatorname{Tr}\left[\rho_{\mathrm{A}} e^{i \mu_{\mathrm{E}} Q_{\mathrm{A}}}\right]$. As we will see below, the analytic continuation between (1.2) and (1.3) is typically straightforward in the vicinity of the origin $\mu=0$, but one typically encounters an interesting structure of singularities along the imaginary $\mu$-axis.

In quantum field theory, Rényi entropies can be evaluated using the replica trick [2], which relates them to a Euclidean path integral on an $n$-sheeted geometry. These 
path integral calculations of $S_{n}$ are easily extended to compute our new charged Rényi entropies. As we will describe below, the key new ingredient is a Wilson line encircling the entangling surface. This generalizes the twist operator $\sigma_{n}$ appearing in the replica trick to include a 'magnetic flux' proportional to $\mu$.

In conformal field theories, these entropies can be studied rather explicitly. A useful approach was introduced in [8] to evaluate the entanglement entropy across a spherical entangling surface for an arbitrary $d$-dimensional CFT in flat space. The latter entropy is related by a conformal mapping to the thermal entropy of the CFT on a hyperbolic cylinder $R \times H^{d-1}$, where the temperature and curvature are fixed by the radius of the original entangling surface. If the temperature is allowed to vary, the thermal entropy calculates Rényi entropies [9, 10]. In theories with holographic gravity duals, the thermal entropy is the horizon entropy of a black hole with $H^{d-1}$ horizon. Our charged entropies $S_{n}(\mu)$ can also be computed for spherical entangling surfaces with a simple extension of this procedure. The same conformal mapping leads to a grand canonical ensemble with chemical potential $\mu$ for the CFT on the hyperbolic cylinder. In the holographic context, the presence of a global symmetry in the boundary CFT leads to a gauge field in the dual gravity theory. $S_{n}(\mu)$ is then related to the entropy of a hyperbolic black hole which is charged under this gauge field.

This paper is organized as follows: In section 2, we will discuss general features of the charged Rényi entropy and outline its computation in CFT. We also describe the various properties of the corresponding twist operators. In section 3, we compute $S_{n}(\mu)$ in holographic CFT's (in spacetime dimensions $d \geq 3$ ) by considering the charged hyperbolic black holes of the dual Einstein-Maxwell theory. We conclude with a discussion and general comments on the properties of the charge Rényi entropy in section 4 . Three appendices are also included. Appendix A describes computations of $\tilde{S}_{n}\left(\mu_{\mathrm{E}}\right)$ in simple free field theories and appendix B gives the holographic computation in $A d S_{3} / C F T_{2}$. The latter case is notable in that the dependence of the charged Rényi entropy on $n$ agrees with the free field theory result. Finally appendix $\mathrm{C}$ contains various details of the holographic calculations, which are used in section 3.

Charged Rényi entropies arose in several recent papers which appeared while this paper was in preparation. First, they were briefly considered in [11, 12]. Charged Rényi entropies appear in [13], which investigates the dynamical evolution of entanglement entropy in two-dimensional CFT's. The supersymmetric Rényi entropies threedimensional $\mathcal{N} \geq 2$ superconformal theores calculated in [14] can be cast in the form (1.3) with an imaginary chemical potential for a circular entangling surface, using the conformal mappings presented in $[8,9]$. In this case, $Q_{\mathrm{A}}$ corresponds to the $R$-charge of the underlying theory. 


\section{Charged Rényi entropies for CFT's}

In this section, we begin with some general comments about the charged Rényi entropies (1.2). We then focus on their computation in conformal field theories, extending the approach of $[8,9]$, to relate charged Rényi entropies for spherical entangling surfaces in flat space to the thermal entropy of the CFT on $R \times H^{d-1}$ in grand canonical ensemble. We also describe the calculation of the conformal weight and magnetic response of the corresponding twist operator. Finally, we describe the computation of these entropies for two-dimensional CFT using twist operators.

\subsection{Replica Trick}

To begin, let us recall the replica trick $[2,3]$. For simplicity, we focus on entanglements in the ground state of a QFT in $d$-dimensional flat space. One begins by introducing an entangling surface $\Sigma$ which divides the spatial slice at time $t_{\mathrm{E}}=0$ into two regions, A and B. ${ }^{1}$ Integer powers of the reduced density matrix $\rho_{\mathrm{A}}$ are represented by a Euclidean path integral

$$
\operatorname{Tr} \rho_{\mathrm{A}}^{n}=Z_{n} /\left(Z_{1}\right)^{n} .
$$

Here $Z_{n}$ is the partition function on an $n$-fold cover of (Euclidean) flat space with cuts introduced on region $\mathrm{A}$ at $t_{\mathrm{E}}=0$. At the cut, copy $n$ is connected to copy $n+1$ when approaching from $t_{\mathrm{E}} \rightarrow 0^{-}$and to copy $n-1$ when approaching from $t_{\mathrm{E}} \rightarrow 0^{+}$. In this construction, the entangling surface $\Sigma$ becomes the branch-point of the branch-cut which separates different copies in the n-fold covering geometry. It is convenient to think of these boundary conditions as produced by the insertion of a $(d-2)$-dimensional surface operator at $\Sigma$, i.e., a twist operator $\sigma_{n}[2] .{ }^{2}$ The factors of $Z_{1}$ appear above to ensure that the density matrix is properly normalized with $\operatorname{Tr}\left[\rho_{\mathrm{A}}\right]=1$. With the usual definition (1.1), the Rényi entropies become

$$
S_{n}=\frac{1}{n-1}\left(n \log Z_{1}-\log Z_{n}\right)
$$

Of course, one would like to consider an analytic continuation to real values of $n$ to determine the entanglement entropy with $S_{\mathrm{EE}}=\lim _{n \rightarrow 1} S_{n}$. Similarly, two other interesting limits are given by $n \rightarrow 0$ and $\infty$, which yield expressions which are known as the Hartley entropy and the min-entropy, respectively [17]. In particular, one finds $S_{0}=\log (\mathbf{d})$ where $\mathbf{d}$ is the number of nonvanishing eigenvalues of $\rho_{\mathrm{A}}$ and $S_{\infty}=$ $-\log \left(\lambda_{1}\right)$ where $\lambda_{1}$ is the largest eigenvalue of $\rho_{\mathrm{A}}$.

\footnotetext{
${ }^{1}$ Implicitly, an initial step in these calculations is to Wick rotate the time coordinate: $t_{\mathrm{E}}=i t$.

${ }^{2}$ For further discussion of twist operators beyond $d=2$, see also [9, 15, 16].
} 
We wish to extend these path integral calculations of $S_{n}$ to compute charged Rényi entropies. Let us first recall how a chemical potential $\mu$ is included in the standard Euclidean path integral representation of a (grand canonical) thermal ensemble. In this framework, the chemical potential is represented by a fixed background gauge potential ${ }^{3}$ $B_{\mu}$ which couples to the relevant conserved current. Of course, in the 'thermal' path integral, the Euclidean time direction is compactified with period $\Delta t_{\mathrm{E}}=1 / T$ and then the chemical potential appears as a nontrivial Wilson line on this thermal circle, i.e., $\oint B=-i \mu / T$.

To evaluate the charged Rényi entropies (1.2), we must compute a grand canonical version of eq. (2.1), i.e.,

$$
\operatorname{Tr}\left[\rho_{\mathrm{A}} \frac{e^{\mu Q_{\mathrm{A}}}}{n_{\mathrm{A}}(\mu)}\right]^{n}=\frac{Z_{n}(\mu)}{\left(Z_{1}(\mu)\right)^{n}} .
$$

with $n_{\mathrm{A}}(\mu) \equiv \operatorname{Tr}\left[\rho_{\mathrm{A}} e^{\mu Q_{\mathrm{A}}}\right]$. Here $Z_{n}(\mu)$ is computed as above, except with the insertion of a Wilson line encircling the entangling surface $\Sigma$. That is, we introduce a fixed background gauge field coupling to the conserved current. This background field is such that loops encircling the entangling surface carry a nontrivial Wilson line, $\oint_{\mathcal{C}} B=$ $-i n \mu .{ }^{4}$ Here the factor $n$ is analogous to the $1 / T$ factor appearing for the thermal ensemble above and arises here because the loop $\mathcal{C}$ circles $n$ times around $\Sigma$, passing through all $n$ sheets of the covering geometry. The Wilson line should be the same on all such curves and so the background gauge field is flat, i.e., $d B=0$, away from the entangling surface. By Stokes' theorem these loops enclose a fixed flux, i.e., $\int_{M} d B=$ $\oint_{\mathcal{C}=\partial M} B=-i n \mu$ for any two-dimensional surface pierced by $\Sigma$. Thus the entangling surface carries a 'magnetic flux' -in $\mu$ of the background gauge field. An alternative perspective is that eq. (2.3) defines a generalized class of twist operators $\tilde{\sigma}_{n}(\mu)$, which are constructed by binding to the original twist operators $\sigma_{n}$, a $(d-2)$-dimensional 'Dirac sheet' carrying the magnetic flux -in $\mu$. Another noteworthy comment is that with the above definitions, the chemical potential in our charged Rényi entropy (2.3) is dimensionless, in contrast to the standard chemical potential in a thermal context, which carries the units of energy. ${ }^{5}$ In any event, given the path integral construction describing eq. (2.3), the charged Rényi entropies (1.2) become

$$
S_{n}(\mu)=\frac{1}{n-1}\left(n \log Z_{1}(\mu)-\log Z_{n}(\mu)\right) .
$$

\footnotetext{
${ }^{3}$ We use $B_{\mu}$ to distinguish this nondynamical gauge field from the bulk gauge potential $A_{\mu}$ appearing in our holographic calculations. This background gauge field is imaginary in the Euclidean path integral, corresponding to a real chemical potential.

${ }^{4}$ The orientation of the contour will become evident in our examples below.

${ }^{5}$ In the thermal context, these units have a natural meaning by comparing the chemical potential to the temperature. In the entanglement context, there is no such natural reference scale with which to compare $\mu$.
} 
Above, we considered a real chemical potential $\mu$, as would appear in standard thermodyanmics. We will also consider analytic continuations of the chemical potential to imaginary values, as in eq. (1.3). As motivation, we note that working with an imaginary chemical potential has proven to be a useful way to probe the confinement phase transition in QCD [18] and to avoid the sign problem in the lattice fermion algorithms [19]. Computations of the Witten index can also be interpreted in a similar fashion [20]. Replacing $\mu=i \mu_{\mathrm{E}}$ in our analysis above, the Wilson loop of the background gauge field now becomes real with $\oint_{\mathcal{C}} B=n \mu_{\mathrm{E}}$. Further the corresponding magnetic flux carried by the generalized twist operators is also real. Hence the effect of the imaginary chemical potential is to introduce a simple phase as a charged operator circles around the entangling surface. Note that the analytic continuation between real and imaginary values requires care because, as we will see below, the partition function has an interesting singularity structure in the complex $\mu$-plane. We will consider both real and imaginary chemical potentials in the following. These two cases will always be distinguished by the notation $\mu$ and $\mu_{\mathrm{E}}$, respectively.

\subsection{Spherical entangling surfaces}

For the remainder of this section we will focus on computations of charged Rényi entropy in $d$-dimensional conformal field theories. We will consider a CFT in flat space in its vacuum state, and choose the entangling surface to be a sphere of radius $R$ (in a constant time slice). In this case, the argument of [8] implies that the usual entanglement entropy equals the thermal entropy of the CFT on a hyperbolic cylinder $R \times H^{d-1}$, where the temperature and curvature are fixed by the radius of the original entangling surface. A simple extension of this approach also allows one to calculate Rényi entropies [9]. We will compute charged Rényi entropies by further extending the procedure to include a background gauge field. For simplicity of notation we will use an imaginary chemical potential, but of course the same construction applies for a real chemical potential.

We begin with a brief review of $[8,9]$. First, we write the metric on flat Euclidean space in terms of a complex coordinate $\omega=r+i t_{\mathrm{E}}$ :

$$
d s_{R^{d}}^{2}=d \omega d \bar{\omega}+\left(\frac{\omega+\bar{\omega}}{2}\right)^{2} d \Omega_{d-2}^{2},
$$

where $t_{\mathrm{E}}$ is the Euclidean time coordinate, $r$ is the radial coordinate on the constant time slices and $d \Omega_{d-2}$ is a standard round metric on a unit $(d-2)$-sphere. The entangling surface is the sphere at $\left(t_{\mathrm{E}}, r\right)=(0, R)$.

We will perform a conformal transformation of the above $R^{d}$ geometry to $H^{d-1} \times S^{1}$

as follows. Introducing a second complex coordinate $\sigma=u+i \frac{\tau_{\mathrm{E}}}{R}$, we perform the 
coordinate transformation, which, in terms of the complex coordinate $\sigma$ defined by

$$
e^{-\sigma}=\frac{R-\omega}{R+\omega}
$$

the metric (2.5) then takes the form

$$
d s_{R^{d}}^{2}=\Omega^{-2} R^{2}\left[d \sigma d \bar{\sigma}+\sinh ^{2}\left(\frac{\sigma+\bar{\sigma}}{2}\right) d \Omega_{d-2}^{2}\right]
$$

where

$$
\Omega=\frac{2 R^{2}}{\left|R^{2}-\omega^{2}\right|}=|1+\cosh \sigma|
$$

The $\Omega^{-2}$ prefactor can now be removed by a simple Weyl rescaling. Letting $\sigma=u+i \frac{\tau_{\mathrm{E}}}{R}$, the resulting conformally transformed metric is

$$
d s_{H^{d-1} \times S^{1}}^{2}=\Omega^{2} d s_{R^{d}}^{2}=d \tau_{\mathrm{E}}^{2}+R^{2}\left(d u^{2}+\sinh ^{2} u d \Omega_{d-2}^{2}\right) .
$$

This is $S^{1} \times H^{d-1} ; u$ is the (dimensionless) radial coordinate on the hyperboloid $H^{d-1}$ and $\tau_{\mathrm{E}}$ is the Euclidean time coordinate on $S^{1}$. The curvature radius of $H^{d-1}$ is $R$, the radius of the original spherical entangling surface. The periodicity of the $\tau_{\mathrm{E}}$ circle is $2 \pi R$. Note that the original entangling surface has been pushed out to the asymptotic boundary, i.e., $u \rightarrow \infty$, in the conformally transformed geometry (2.9).

The key point is that, under this conformal mapping, the density matrix describing the CFT vacuum state on the interior of the entangling surface is transformed to a thermal density matrix with temperature

$$
T_{0}=\frac{1}{2 \pi R}
$$

on the new hyperbolic geometry. That is, the reduced density matrix related to the thermal density matrix as

$$
\rho_{\mathrm{A}}=U^{-1} \frac{e^{-H / T_{0}}}{Z\left(T_{0}\right)} U,
$$

where $U$ is the unitary transformation implementing the conformal transformation. Since the entropy is insensitive to unitary transformations, the desired entanglement entropy just equals the thermal entropy in the transformed space. This same conformal mapping can also be used to evaluate the Rényi entropy. The only difference is that it would be applied to the $n$-fold cover of flat space used to evaluate eq. (2.1). In this case the period of $\tau_{\mathrm{E}}$ is $2 \pi R n$, so the corresponding thermal ensemble has a temperature $T=T_{0} / n$. 
We can compute charged Rényi entropies (1.3) by generalizing this approach. First after having identified the appropriate charge $Q$, we introduce a (dimensionless) chemical potential $\mu$ and the previous density matrix (2.11) becomes

$$
\rho_{\text {therm }}=\frac{e^{-H / T_{0}+\mu Q}}{Z\left(T_{0}, \mu\right)} .
$$

Now, in fact, our discussion is slightly simplified if we consider instead an imaginary chemical potential $\mu_{\mathrm{E}}=-i \mu$ with which the above expression turns into

$$
\rho_{\text {therm }}=\frac{e^{-H / T_{0}+i \mu_{\mathrm{E}} Q}}{Z\left(T_{0}, \mu_{\mathrm{E}}\right)} .
$$

As discussed above, this chemical potential is incorporated into the thermal path integral via a background gauge field with a nontrivial Wilson line on the Euclidean time circle:

$$
\mu_{\mathrm{E}}=\oint B=\int_{0}^{2 \pi R} B_{\tau_{\mathrm{E}}} d \tau_{\mathrm{E}} .
$$

In this case the potential is just constant: $B_{\tau_{\mathrm{E}}}=\mu_{\mathrm{E}} /(2 \pi R)$. The background gauge field is invariant under the conformal transformation mapping between the hyperbolic geometry and flat space. Therefore in the flat space coordinates, we may express this gauge field as

$$
\begin{aligned}
B & =\frac{i R}{2 \pi} \mu_{\mathrm{E}}\left[\frac{d \omega}{R^{2}-\omega^{2}}-\frac{d \bar{\omega}}{R^{2}-\bar{\omega}^{2}}\right] \\
& =-\frac{R}{\pi} \mu_{\mathrm{E}} \frac{2 t_{\mathrm{E}} r d r+\left(R^{2}-r^{2}+t_{\mathrm{E}}^{2}\right) d t_{\mathrm{E}}}{\left(R^{2}-r^{2}+t_{\mathrm{E}}^{2}\right)^{2}+4 t_{\mathrm{E}}^{2} r^{2}} .
\end{aligned}
$$

Now one can readily verify that this background gauge field yields $\oint B=\mu_{\mathrm{E}}$ for any contour encircling the entangling surface at $\left(t_{\mathrm{E}}, r\right)=(0, R)$ in the flat space geometry. ${ }^{6}$ Of course, we also have $d B=0$ and so this is precisely the background required to evaluate the charged Rényi entropy (1.3) in this particular case.

Hence we must simply supplement the conformal mapping approach of [8, 9] with the background gauge field (2.15) to evaluate the charged Rényi entropy across a spherical entangling surface for the CFT vacuum in flat space. The effective reduced density matrix in eq. (1.3) is again simply related to the thermal density matrix (2.13) on the hyperbolic space:

$$
\rho_{\mathrm{A}} \frac{e^{i \mu_{\mathrm{E}} Q_{\mathrm{A}}}}{\tilde{n}_{\mathrm{A}}\left(\mu_{\mathrm{E}}\right)}=U^{-1} \rho_{\text {therm }} U=U^{-1} \frac{e^{-H / T_{0}+i \mu_{\mathrm{E}} Q}}{Z\left(T_{0}, \mu_{\mathrm{E}}\right)} U,
$$

\footnotetext{
${ }^{6}$ An interesting exercise to gain better intuition for this background gauge field (2.15) is to expand the coordinates near the spherical entangling surface: $t_{\mathrm{E}}=\rho \sin \theta$ and $r=R+\rho \cos \theta$ with $\rho \ll R$. To leading order in $\rho / R$, one then finds that the potential reduces to $B \simeq \frac{\mu_{\mathrm{E}}}{2 \pi} d \theta$.
} 
where as in eq. (2.11), $U$ is the unitary transformation implementing the conformal transformation between the two geometries. Given this expression, the charged Rényi entropy (1.3) can be evaluated in terms of the corresponding thermal partition function for the CFT on the hyperbolic geometry as

$$
\tilde{S}_{n}\left(\mu_{\mathrm{E}}\right)=\frac{1}{1-n} \log \frac{Z\left(T_{0} / n, \mu_{\mathrm{E}}\right)}{Z\left(T_{0}, \mu_{\mathrm{E}}\right)^{n}}
$$

Recall that in evaluating $Z\left(T_{0} / n, \mu_{\mathrm{E}}\right)$, the period of $\tau_{\mathrm{E}}$ is extended to $2 \pi R n$, however, the gauge potential remains fixed as $B_{\tau_{\mathrm{E}}}=\mu_{\mathrm{E}} /(2 \pi R)$. Hence the total Wilson line around the thermal circle increases by a factor of $n$ as desired, i.e., $\oint B=n \mu_{\mathrm{E}}$. Now using the standard thermodynamic identity for the grand canonical ensemble

$$
S_{\text {therm }}\left(T, \mu_{\mathrm{E}}\right)=-\left.\frac{\partial F\left(T, \mu_{\mathrm{E}}\right)}{\partial T}\right|_{\mu_{\mathrm{E}}}=\left.\frac{\partial}{\partial T}\left(T \log Z\left(T, \mu_{\mathrm{E}}\right)\right)\right|_{\mu_{\mathrm{E}}},
$$

one easily derives the following relation between the charged Rényi entropy and the thermal entropy [9]:

$$
\tilde{S}_{n}\left(\mu_{\mathrm{E}}\right)=\frac{n}{n-1} \frac{1}{T_{0}} \int_{T_{0} / n}^{T_{0}} S_{\text {therm }}\left(T, \mu_{\mathrm{E}}\right) d T .
$$

At this point, we may remind the reader that the above discussion makes no reference to the AdS/CFT correspondence. In the special case of a holographic CFT, this analysis may be further extended by evaluating the thermal entropy on the hyperbolic background in terms of the horizon entropy of a topological black hole in the bulk with a hyperbolic horizon [8, 9] — see also [21]. In the context of the charged Rényi entropy (1.2), the boundary CFT also contains a conserved current corresponding to the charge probed by these entropies and hence the bulk theory will also include a dual gauge field. The holographic representation of the grand canonical ensemble considered above will then be a topological black hole which is charged under this gauge field. We will turn to such holographic calculations in section 3. First, however, we continue below with some further remarks which apply to general CFT's.

\subsection{Properties of generalized twist operators}

As discussed at the beginning of this section, the calculation of (either ordinary or charged) Rényi entropies can be viewed as involving the insertion of a twist operator at the entangling surface. A generalized notion of conformal dimension can be defined for these surface operators by considering the leading singularity in the correlator $\left\langle T_{\mu \nu} \sigma_{n}\right\rangle$. This leading singularity is fixed by symmetry, as well as the tracelessness and conservation of the stress tensor. To be precise, consider inserting the stress tensor $T_{\mu \nu}$ at 
a perpendicular distance $y$ from the twist operator $\sigma_{n}$, such that $y$ is much smaller than any scales defining the geometry of the entangling surface $\Sigma$. Then the leading singularity takes the following form ${ }^{7}$

$$
\begin{aligned}
\left\langle T_{a b} \sigma_{n}\right\rangle & =-\frac{h_{n}}{2 \pi} \frac{\delta_{a b}}{y^{d}}, \quad\left\langle T_{a i} \sigma_{n}\right\rangle=0, \\
\left\langle T_{i j} \sigma_{n}\right\rangle & =\frac{h_{n}}{2 \pi} \frac{(d-1) \delta_{i j}-d n_{i} n_{j}}{y^{d}},
\end{aligned}
$$

where $a, b(i, j)$ denote tangential (normal) directions to the twist operator and $n_{i}$ is the unit vector directed orthogonally from the twist operator to the $T_{\mu \nu}$ insertion. Thus the singularity is completely fixed up to the constant $h_{n}$, which is referred to as the conformal dimension of $\sigma_{n}$. The approach reviewed in the previous section can be applied to determine the value of $h_{n}$ in terms of the thermal energy density $\mathcal{E}(T, \mu)$ on the hyperbolic cylinder $[9], 8$

$$
h_{n}(\mu)=\frac{2 \pi n}{d-1} R^{d}\left(\mathcal{E}\left(T_{0}, \mu=0\right)-\mathcal{E}\left(T_{0} / n, \mu\right)\right) .
$$

The first term above arises because of the anomalous behaviour of the stress tensor under conformal transformations. Of course, as is implicit above, these remarks apply equally well for the original twist operators $\sigma_{n}$ and for the generalized twist operators $\tilde{\sigma}_{n}(\mu)$ appearing in the calculation of the charged Rényi entropy. In particular, the arguments of [9] yielding eq. (2.21) apply without any change in the presence of the background gauge potential.

In the context of the charged Rényi entropies, another operator in the underlying CFT is the current $J_{\mu}$, associated with the global charge appearing in eq. (1.2), i.e.,

$$
Q_{\mathrm{A}}=\int_{A} d^{d-1} x J_{t}
$$

Again, symmetries and conservation of the current dictate the form of the leading singularity in the correlator $\left\langle J_{\mu} \tilde{\sigma}_{n}(\mu)\right\rangle$. In this case, the singularity takes the form ${ }^{9}$

$$
\left\langle J_{i} \tilde{\sigma}_{n}(\mu)\right\rangle=\frac{i k_{n}(\mu)}{2 \pi} \frac{\epsilon_{i j} n^{j}}{y^{d-1}}, \quad\left\langle J_{a} \sigma_{n}\right\rangle=0
$$

\footnotetext{
${ }^{7}$ These correlators (2.20) should be normalized by dividing by $\left\langle\sigma_{n}\right\rangle$. However, we leave this implicit to avoid further clutter.

${ }^{8}$ Note that in the Euclidean background, $\mathcal{E}(T, \mu)=-\left\langle T_{\tau_{\mathrm{E}} \tau_{\mathrm{E}}}\right\rangle$. Also observe that we phrase the discussion in this subsection in terms of a real chemical potential $\mu$.

${ }^{9}$ Note that the correlators here and in eq. (2.20) are implicitly evaluated in the Euclidean path integral.
} 
where $\epsilon_{i j}$ is the volume form in the two-dimensional space transverse to $\Sigma$. This parityodd tensor appears in the correlator because of the magnetic flux carried by the generalized twist operator. We refer to $k_{n}$ as the 'magnetic response,' since this parameter characterizes the response of the current to the magnetic flux.

Following [9], we can determine the value of $k_{n}$ using the conformal mapping in the above discussion of spherical entangling surfaces. In this case, one begins with the charge density that appears in the grand canonical ensemble on the hyperbolic cylinder: $\left\langle J_{\tau_{\mathrm{E}}}\right\rangle=-i \rho(n, \mu)$. Now conformally mapping to the $n$-fold cover of $R^{d}$, this expectation value becomes

$$
\left\langle J_{\mu} \tilde{\sigma}_{n}(\mu)\right\rangle_{\text {flat }}=\Omega^{d-2} \frac{\partial X^{\alpha}}{\partial Y^{\mu}}\left\langle J_{\alpha}\right\rangle_{\text {hyperbolic }} .
$$

The form of the transformation is fixed because the current has conformal dimension $d-1{ }^{10}$ Now as indicated on the left-hand side of eq. (2.24), this mapping yields the correlator of the current with the spherical twist operator. Further, taking the limit where the current insertion approaches the twist operator, one recovers the leading singularity in eq. (2.23). Hence using eqs. (2.6) and (2.8), the magnetic response can be evaluated as

$$
k_{n}(\mu)=2 \pi n R^{d-1} \rho(n, \mu) .
$$

Here, the additional factor of $n$ appears because the correlators in eq. (2.23) are understood to involve the the total current for the entire $n$-fold replicated CFT whereas eq. (2.24) corresponds to the insertion of $J_{\mu}$ on a single sheet of the $n$-fold cover. Hence we must multiply by an extra factor of $n$ to compare the two expressions.

An interesting universal property of $h_{n}$ was obtained for higher dimensional twist operators in $[9,16]$ (see also [22]):

$$
\left.\partial_{n} h_{n}\right|_{n=1}=2 \pi^{\frac{d}{2}+1} \frac{\Gamma(d / 2)}{\Gamma(d+2)} C_{T} .
$$

Here $C_{T}$ is the central charge defined by the two-point function of the stress tensor ${ }^{11}$

$$
\left\langle T_{\mu \nu}(x) T_{\rho \sigma}(0)\right\rangle=\frac{C_{T}}{x^{2 d}} \mathcal{I}_{\mu \nu, \rho \sigma}
$$

\footnotetext{
${ }^{10}$ One can also verify that this transformation (2.24) ensures that the charge operator (2.22) defined on the interior of the sphere, i.e., $Q_{\mathrm{A}}=i \int_{t_{\mathrm{E}}=0, r<R} d^{d-1} x J_{t_{\mathrm{E}}}$, is just the conformal transformation of the charge defined by integrating $J_{\tau_{\mathrm{E}}}$ over the entire hyperbolic plane $H^{d-1}$ - as is implicit in our discussions above.

${ }^{11}$ Note that our normalization for $C_{T}$ here is a standard one but it is not the same as in [9]. Hence the numerical factors in eq. (2.26) are slightly different than in that reference.
} 
where

$$
\mathcal{I}_{\mu \nu, \rho \sigma}=\frac{1}{2}\left(I_{\mu \rho} I_{\nu \sigma}+I_{\mu \sigma} I_{\nu \rho}\right)-\frac{1}{d} \delta_{\mu \nu} \delta_{\rho \sigma} \quad \text { with } \quad I_{\mu \nu}(x)=\delta_{\mu \nu}-2 \frac{x^{\mu} x^{\nu}}{|x|^{2}} .
$$

In fact, similar universal properties is also found for higher derivatives of $h_{n}$ in the vicinity of $n=1$.

The above universal behaviour does not immediately extend to the conformal weight of the generalized twist operators $\tilde{\sigma}(\mu)$. Instead, the natural extension involves an expansion about both $n=1$ and $\mu=0$, as follows:

$$
h_{n}(\mu)=\sum_{a, b} \frac{1}{a ! b !} h_{a b}(n-1)^{a} \mu^{b}
$$

where we defined the coefficients

$$
\left.h_{a b} \equiv\left(\partial_{n}\right)^{a}\left(\partial_{\mu}\right)^{b} h_{n}(\mu)\right|_{n=1, \mu=0} .
$$

Note that the twist operator becomes trivial when $n=1$ and $\mu=0$ and hence the first term in this expansion vanishes, i.e., $h_{00}=0$. Further $h_{10}=\left.\partial_{n} h_{n}(\mu)\right|_{n=1, \mu=0}$ is precisely the term appearing in eq. (2.26). Now recall the expression (2.21) for the weight, which we rewrite as

$$
h_{n}(\mu)=\frac{2 \pi n}{d-1} R^{d}\left(\left.\left\langle T_{\tau_{\mathrm{E}} \tau_{\mathrm{E}}}\right\rangle\right|_{T_{0} / n, \mu}-\left.\left\langle T_{\tau_{\mathrm{E}} \tau_{\mathrm{E}}}\right\rangle\right|_{T_{0}, \mu=0}\right) .
$$

in terms of the Euclidean stress tensor. Here both expectation values are in the grand canonical ensemble on the hyperbolic space. That is, in terms of the thermal density matrix given in eq. (2.12), the density matrix determining the second expectation value is $\rho_{\text {therm }}(\mu=0)$ while that in the first is $\left[\rho_{\text {therm }}\right]^{n}$. Now we can produce the same double expansion as in eq. (2.29) by re-expressing the latter with

$$
\left[e^{-H / T_{0}+\mu Q}\right]^{n}=e^{-H / T_{0}}\left[e^{-(n-1) H / T_{0}+n \mu Q}\right]
$$

and expanding the last factor in terms of $n-1$ and $\mu[16,22]$. Here, we note that the manipulation in eq. (2.32) is valid since $Q$ is a conserved charge and hence $[H, Q]=0$. Now it is straightforward to show that the expansion coefficients $h_{a b}$ in eq. (2.30) are given by

$$
h_{a b}=\left.\frac{2 \pi}{d-1} R^{d}\left(\partial_{n}\right)^{a}\left(\partial_{\mu}\right)^{b}\left(n \frac{Z\left(T_{0}, \mu=0\right)}{Z\left(T_{0} / n, \mu\right)}\left\langle T_{\tau_{\mathrm{E}} \tau_{\mathrm{E}}} e^{-(n-1) H / T_{0}+n \mu Q}\right\rangle-n\left\langle T_{\tau_{\mathrm{E}} \tau_{\mathrm{E}}}\right\rangle\right)\right|_{n=1, \mu=0}
$$


Note that both of the expectation values above are evaluated in the thermal ensemble with $T=T_{0}$ and $\mu=0$. Hence these coefficients can be determined in terms of correlators of the stress tensor and the conserved current in the thermal bath on the hyperbolic geometry at temperature $T_{0}$. However, by applying the conformal transformation, these correlators may also be evaluated for the CFT vacuum in flat space.

Let us focus here on the corrections to the conformal dimension of the twist operator coming from a small chemical potential $\mu$ at $n=1$, i.e.,

$$
h_{0 b}=\frac{2 \pi R^{d}}{d-1} i^{b}\left\langle T_{\tau_{\mathrm{E}} \tau_{\mathrm{E}}} \prod_{i=1}^{b} \int_{H^{d-1}} d^{d-1} \sigma_{i} J_{\tau_{\mathrm{E}}}\left(\sigma_{i}\right)\right\rangle_{c}
$$

where the subscript $c$ denotes the connected correlator. Again, this correlator is evaluated in the thermal ensemble on the hyperbolic space with $T=T_{0}$ and $\mu=0$. However, as noted above, it is convenient to transform back to flat space where the correlators will be evaluated in the CFT vacuum. First, however, we observe that if we evaluate the correlator in eq. (2.34) with a Euclidean path integral on $S^{1} \times H^{d-1}$, then the stress tensor maybe inserted at any position in this background. Hence we choose to place $T_{\tau_{\mathrm{E}} \tau_{\mathrm{E}}}$ at $\left(\tau_{\mathrm{E}}, \sigma\right)=(\pi R, 0)$ which the conformal transformation then maps to $\left(t_{\mathrm{E}}, r\right)=(\infty, 0)$ in the corresponding flat space background. To be precise, with this choice, the conformal transformation yields the following simple expression:

$$
T_{\tau_{\mathrm{E}} \tau_{\mathrm{E}}}=\lim _{t_{\mathrm{E}} \rightarrow \infty}\left(\frac{t_{\mathrm{E}}^{2}}{2 R^{2}}\right)^{d} T_{t_{\mathrm{E}} t_{\mathrm{E}}}
$$

and so eq. (2.34) becomes

$$
h_{0 b}=\frac{2 \pi R^{d}}{d-1} i^{b} \lim _{t_{\mathrm{E}} \rightarrow \infty}\left(\frac{t_{\mathrm{E}}^{2}}{2 R^{2}}\right)^{d}\left\langle T_{t_{\mathrm{E}} t_{\mathrm{E}}} \prod_{i=1}^{b} \int_{r<R} d^{d-1} x_{i} J_{t_{\mathrm{E}}}\left(x_{i}\right)\right\rangle_{c}
$$

One immediate observation is that in the CFT vacuum in flat space, the two-point correlator $\langle T J\rangle$ will vanish and hence we must have $h_{01}=0$. That is, the linear correction in $\mu$ to the conformal weight of the twist operator vanishes at $n=1$.

Hence the leading contribution should appear at order $\mu^{2}$ and is determined by the three-point correlator $\langle T J J\rangle$. The latter correlation function has a universal form dictated by conformal symmetry, up to a few constants which are determined by the underlying CFT [23]. Therefore $h_{02}$ can be determined entirely in terms of these few parameters. More explicitly, the $\langle T J J\rangle$ correlator takes the following form in a $d$ dimensional CFT [23]

$$
\left\langle T_{\mu \nu}\left(x_{1}\right) J_{\gamma}\left(x_{2}\right) J_{\delta}\left(x_{3}\right)\right\rangle=\frac{t_{\mu \nu \alpha \beta}\left(\mathbf{X}_{\mathbf{2 3}}\right) I_{\gamma}{ }^{\alpha}\left(x_{21}\right) I_{\delta}^{\beta}\left(x_{31}\right)}{\left|x_{12}\right|^{d}\left|x_{13}\right|^{d}\left|x_{23}\right|^{d-2}},
$$


where

$$
\mathbf{x}_{12}=\mathbf{x}_{1}-\mathbf{x}_{2}, \quad \mathbf{X}_{23}=\frac{\mathbf{x}_{21}}{\left|x_{21}\right|^{2}}-\frac{\mathbf{x}_{31}}{\left|x_{31}\right|^{2}}, \quad \hat{\mathbf{X}}=\frac{\mathbf{X}}{|\mathbf{X}|},
$$

where $|\mathbf{x}|$ is the norm of the vector. Recall that $I_{\mu \nu}(x)$ was defined in eq. (2.28) and further we have

$$
\begin{aligned}
t_{\mu \nu \rho \sigma}(\mathbf{X}) & =\hat{a} h_{\mu \nu}^{1}(\hat{\mathbf{X}}) \delta_{\rho \sigma}+\hat{b} h_{\mu \nu}^{1}(\hat{\mathbf{X}}) h_{\rho \sigma}^{1}(\hat{\mathbf{X}})+\hat{c} h_{\mu \nu \rho \sigma}^{2}(\hat{\mathbf{X}})+\hat{e} h_{\mu \nu \rho \sigma}^{3}(\hat{\mathbf{X}}), \\
h_{\mu \nu}^{1}(\hat{\mathbf{X}})= & \hat{X}^{\mu} \hat{X}^{\nu}-\frac{1}{d} \delta_{\mu \nu}, \\
h_{\mu \nu \rho \sigma}^{2}(\hat{\mathbf{X}})= & \hat{X}^{\mu} \hat{X}^{\rho} \delta_{\nu \sigma}+\{\mu \leftrightarrow \nu, \rho \leftrightarrow \sigma\} \\
& \quad-\frac{4}{d} \hat{X}^{\mu} \hat{X}^{\nu} \delta_{\rho \sigma}-\frac{4}{d} \hat{X}^{\rho} \hat{X}^{\sigma} \delta_{\mu \nu}+\frac{4}{d^{2}} \delta_{\mu \nu} \delta_{\rho \sigma}, \\
h_{\mu \nu \rho \sigma}^{3}(\hat{\mathbf{X}})= & \delta_{\mu \rho} \delta_{\nu \sigma}+\delta_{\mu \sigma} \delta_{\nu \rho}-\frac{2}{d} \delta_{\mu \nu} \delta_{\rho \sigma} .
\end{aligned}
$$

The coefficients $\hat{a}, \hat{b}, \hat{c}, \hat{e}$ are the parameters characterizing the underlying CFT. However, only two of these constants are independent as they satisfy the following constraints [23]:

$$
d \hat{a}-2 \hat{b}+2(d-2) \hat{c}=0, \quad \hat{b}-d(d-2) \hat{e}=0 .
$$

Notice that in the special case $d=2$, both $\hat{a}$ and $\hat{b}$ vanish.

Now we only need to consider the correlator $\left\langle T_{t_{\mathrm{E}} t_{\mathrm{E}}}\left(x_{1}\right) J_{t_{\mathrm{E}}}\left(x_{2}\right) J_{t_{\mathrm{E}}}\left(x_{3}\right)\right\rangle$ in the limit that $x_{1}^{0} \equiv \chi \rightarrow \infty, x_{1}^{i}=0$, while $x_{2}^{0}=x_{3}^{0}=0$ and $\left|x_{2}\right|,\left|x_{3}\right|<R$. To leading order in $\chi$, we find

$$
I_{00}=-1+\cdots, \quad I_{i j}=\delta_{i j}+\cdots, \quad I_{i 0}=\mathcal{O}(1 / \chi)
$$

This immediately implies that for $\mu=\nu=\gamma=\delta=0$ in eq. (2.37), we need only consider $t_{0000}$ to leading order and further we have

$$
t_{0000} \rightarrow \frac{1}{d^{2}}(-d \hat{a}+\hat{b}+4 \hat{c}+2 d(d-1) \hat{e})=\frac{2}{d} \hat{c}+\hat{e}
$$

Then for $h_{02}$ in eq. (2.36), we are left with

$$
\begin{gathered}
\left\langle T_{00}\left(x_{1}^{0} \rightarrow \infty\right) \int_{\left|x_{2}\right|<R} d^{d-1} x_{2} J_{0}\left(x_{2}\right) \int_{\left|x_{3}\right|<R} d^{d-1} x_{3} J_{0}\left(x_{3}\right)\right\rangle \\
=\int_{\left|x_{2}\right|<R} d^{d-1} x_{2} \int_{\left|x_{3}\right|<R} d^{d-1} x_{3} \frac{\frac{2}{d} \hat{c}+\hat{e}}{x_{1}^{2 d}\left|x_{23}\right|^{d-2}} .
\end{gathered}
$$

The integral can be evaluated exactly. Using equation (2.36), we finally arrive at

$$
h_{02}=-\frac{4 \pi^{d-1}}{\Gamma(d+1)}\left(\frac{2}{d} \hat{c}+\hat{e}\right) .
$$


Now an analogous double expansion about $n=1$ and $\mu=0$ can also be applied to the magnetic response:

$$
k_{n}(\mu)=\sum_{a, b} \frac{1}{a ! b !} k_{a b}(n-1)^{a} \mu^{b}
$$

where we defined the coefficients

$$
\left.k_{a b} \equiv\left(\partial_{n}\right)^{a}\left(\partial_{\mu}\right)^{b} k_{n}(\mu)\right|_{n=1, \mu=0} .
$$

Next we recall the expression (2.25), which we rewrite in terms of the Euclidean current as

$$
k_{n}(\mu)=\left.2 \pi i n R^{d-1}\left\langle J_{\tau_{\mathrm{E}}}\right\rangle\right|_{T_{0} / n, \mu} .
$$

Again this expectation value is in the grand canonical ensemble on the hyperbolic space. Now following the same manipulations of the corresponding density matrix as in eq. (2.32), we arrive at the following expressions for $k_{a b}$

$$
k_{a b}=\left.2 \pi i R^{d-1}\left(\partial_{n}\right)^{a}\left(\partial_{\mu}\right)^{b}\left(n \frac{Z\left(T_{0}, \mu=0\right)}{Z\left(T_{0} / n, \mu\right)}\left\langle J_{\tau_{\mathrm{E}}} e^{-(n-1) H / T_{0}+n \mu Q}\right\rangle\right)\right|_{n=1, \mu=0}
$$

where the remaining expectation value above is evaluated in the thermal ensemble with $T=T_{0}$ and $\mu=0$. Hence these coefficients can again be determined in terms of correlators of the stress tensor and the conserved current in the thermal bath on the hyperbolic geometry at temperature $T_{0}$. However, by conformally mapping to flat space, the correlators may alternatively be evaluated in the CFT vacuum.

Let us evaluate a few coefficients for the low order contributions in the expansion (2.46). First, let us note that the coefficient $k_{10}$ will determined in terms of the twopoint correlator $\langle J T\rangle$ and so upon mapping this correlator back to flat space, we will find a vanishing result, i.e., $k_{10}=0$. Considering the next two coefficients, eq. (2.49) yields

$$
\begin{aligned}
& k_{01}=2 \pi i R^{d-1}\left\langle J_{\tau_{\mathrm{E}}} Q\right\rangle_{c}, \\
& k_{11}=-2 \pi i R^{d-1}\left(\frac{1}{T_{0}}\left\langle J_{\tau_{\mathrm{E}}} Q H\right\rangle_{c}-2\left\langle J_{\tau_{\mathrm{E}}} Q\right\rangle_{c}\right),
\end{aligned}
$$

where subscript $c$ again denotes the connected correlators. These correlators can be evaluated following the approach described above in evaluating $h_{02}$. In particular, we conformally map these expressions back to flat space after making a judicious choice for the position of the current insertion. The resulting three-point function in eq. (2.51) 
can be evaluated using the $\langle T J J\rangle$ correlator given in eq. (2.37). Similarly, the twopoint function appearing in both expressions can be evaluated using the current-current correlator

$$
\left\langle J_{\mu}(x) J_{\nu}(0)\right\rangle=\frac{C_{V}}{x^{2(d-1)}} I_{\mu \nu}(x),
$$

where $I_{\mu \nu}(x)$ was defined in eq. (2.28). Note that a Ward identity relates the constant $C_{V}$ to the parameters appearing in the three-point correlator (2.37) with [23]

$$
C_{V}=\frac{2 \pi^{d / 2}}{\Gamma\left(\frac{d+2}{2}\right)}(\hat{c}+\hat{e}) .
$$

Without discussing the calculations in more detail, let us present the following results

$$
\begin{aligned}
\left\langle J_{\tau_{\mathrm{E}}} Q\right\rangle_{c} & =-i \frac{\pi^{(d-1) / 2}}{2^{d-2}(d-1) \Gamma((d-1) / 2)} \frac{C_{V}}{R^{d-1}}, \\
\left\langle J_{\tau_{\mathrm{E}}} Q H\right\rangle_{c} & =-i \frac{2 \pi^{d-2}}{d \Gamma(d-1)} \frac{1}{R^{d}}\left(\frac{2}{d} \hat{c}+\hat{e}\right) .
\end{aligned}
$$

Substituting these results (2.54), as well as eq. (2.53), into eqs. (2.50) and (2.51) then yields

$$
\begin{aligned}
& k_{01}=\frac{8 \pi^{d}}{\Gamma(d+1)}(\hat{c}+\hat{e}), \\
& k_{11}=\frac{8 \pi^{d}}{d \Gamma(d+1)}(2 \hat{c}-d(d-3) \hat{e}) .
\end{aligned}
$$

We might re-express the result for $k_{01}$ in a form similar to that appearing in eq. (2.26) for the conformal weight, namely,

$$
\left.\partial_{\mu} k_{n}(\mu)\right|_{n=1, \mu=0}=4 \pi^{d / 2} \frac{\Gamma\left(\frac{d+2}{2}\right)}{\Gamma(d+1)} C_{V},
$$

where $C_{V}$ is the central charge appearing in the current-current correlator (2.52).

\subsection{Generalized twist operators in $d=2$}

In this subsection, we compute charged Rényi entropies using twist operators in a simple two-dimensional CFT. ${ }^{12}$ In particular, we consider a free massless Dirac fermion $\psi$ on an infinite line and we are interested in the Rényi entropy of a subsystem $x \in[u, v]$. In accord with the review at the beginning of this section, the Rényi entropy can

\footnotetext{
${ }^{12}$ The analysis in this section was first done by T. Takayanagi [24]. We thank him for sharing these results with us.
} 
determined by evaluating the partition function of $\psi$ on an $n$-sheeted cover of $R^{2}$, which is equivalent to the correlation function of twist operators inserted at the entangling surface, i.e., the two points $x=u, v[2]$. Let us first review the computation of the free fermion without the Wilson loop, as in [25]. On a $n$-fold cover, there is a branch cut connecting $x=u$ and $v$ and each time we cross the branch cut, we change from one sheet to the next. Let us label the fermion on $k$-th sheet as $\psi_{k}$, where $k$ runs from 1 to $n$. Then the fields on the different sheets are identified as follows:

$$
\psi_{k}\left(e^{2 \pi i}(w-u)\right)=\psi_{k+1}(w-u), \quad \psi_{k}\left(e^{2 \pi i}(w-v)\right)=\psi_{k-1}(w-v)
$$

where we used the complexified coordinate $w=x+i t_{\mathrm{E}}$. These boundary conditions can be 'diagonalized' by defining $n$ new fields

$$
\tilde{\psi}_{m}=\frac{1}{n} \sum_{k=1}^{n} e^{2 \pi i k m / n} \psi_{k}
$$

for which the boundary conditions (2.58) become

$$
\tilde{\psi}_{m}\left(e^{2 \pi i}(w-u)\right)=e^{2 \pi i m / n} \tilde{\psi}_{m}(w-u), \tilde{\psi}_{m}\left(e^{2 \pi i}(w-v)\right)=e^{-2 \pi i m / n} \tilde{\psi}_{m}(w-v)(2.6
$$

where $m=-(n-1) / 2,-(n-1) / 2+1, \cdots,(n-1) / 2$. The phase shifts in eq. (2.60) are generated by standard twist operators $\sigma_{m / n}$, each of which act only on the corresponding $\tilde{\psi}_{m}$ and which have conformal dimension $\Delta_{m}=\frac{1}{2}(m / n)^{2}$. The full twist operator $\sigma_{n}$ appearing in evaluating the Rényi entropy can then be written as $\sigma_{n}=\prod \sigma_{m / n}$ and hence the desired correlator of the twist operators $\sigma_{n}$ and $\sigma_{-n}$ yields:

$$
Z_{n}=\left\langle\sigma_{n}(u) \sigma_{-n}(v)\right\rangle=\prod_{m=-\frac{n-1}{2}}^{\frac{n-1}{2}}\left\langle\sigma_{m / n}(u) \sigma_{-m / n}(v)\right\rangle \sim|u-v|^{-4 \Delta_{n}}
$$

where total conformal dimension $\Delta_{n}$ appearing above is given by

$$
\Delta_{n}=\sum_{m=-\frac{n-1}{2}}^{\frac{n-1}{2}} \frac{1}{2}\left(\frac{m}{n}\right)^{2}=\frac{1}{24}\left(n-\frac{1}{n}\right)
$$

Then applying eq. (2.2) to evaluate the Rényi entropy, we recover the well-known result

$$
S_{n}=\frac{1}{6}\left(1+\frac{1}{n}\right) \log |u-v|
$$

We now generalize the above discussion to evaluate the charged Rényi entropy. In particular, the charge, which we consider here, will be that associated with global phase 
rotations of the fermion, $\psi \rightarrow e^{i \theta} \psi$. If we consider an imaginary chemical potential $\mu_{\mathrm{E}}$, the effect of the Wilson loop is easily represented by extending the original boundary conditions (2.58) to include a additional phase:

$$
\psi_{k}\left(e^{2 \pi i}(w-u)\right)=e^{i \mu_{\mathrm{E}}} \psi_{k+1}(w-u), \quad \psi_{k}\left(e^{2 \pi i}(w-v)\right)=e^{-i \mu_{\mathrm{E}}} \psi_{k-1}(w-v)
$$

Since this additional phase is added uniformly, the 'diagonal' fields (2.59) now satisfy

$$
\begin{aligned}
\tilde{\psi}_{m}\left(e^{2 \pi i}(w-u)\right) & =e^{2 \pi i m / n+i \mu_{\mathrm{E}}} \tilde{\psi}_{m}(w-u), \\
\tilde{\psi}_{m}\left(e^{2 \pi i}(w-v)\right) & =e^{-2 \pi i m / n-i \mu_{\mathrm{E}}} \tilde{\psi}_{m}(w-v) .
\end{aligned}
$$

These phase shifts are accomplished by introducing twist operators, $\sigma_{\alpha\left(m, \mu_{\mathrm{E}}\right)}$ and $\sigma_{-\alpha\left(m, \mu_{\mathrm{E}}\right)}$, where

$$
\alpha\left(m, \mu_{\mathrm{E}}\right)=\frac{m}{n}+\frac{\mu_{\mathrm{E}}}{2 \pi}+\ell_{m},
$$

where $m$ runs from $-\frac{n-1}{2}$ to $\frac{n-1}{2}$ as, before. The conformal dimension of these twist operators is now

$$
\Delta_{\alpha\left(m, \mu_{\mathrm{E}}\right)}=\frac{1}{2} \alpha\left(m, \mu_{\mathrm{E}}\right)^{2}=\frac{1}{2}\left(\frac{m}{n}+\frac{\mu_{\mathrm{E}}}{2 \pi}+\ell_{m}\right)^{2} .
$$

The constant $\ell_{m}$ appearing above is an integer which is chosen to minimize the conformal dimension of the corresponding twist operator. This freedom arises because of the ambiguity in defining the phase factors in eq. (2.65) modulo $2 \pi$. For example, shifting $\ell_{m}$ from 0 to 1 changes the corresponding phase factor by $2 \pi$ and so leaves the corresponding boundary condition in eq. (2.65) unchanged. The conformal dimension (2.67) is always minimized by choosing $\ell_{m}$ so that the phase factor generated by the twist operator lies between $-\pi$ and $\pi$, i.e., such that $-\frac{1}{2} \leq \alpha\left(m, \mu_{\mathrm{E}}\right) \leq \frac{1}{2}$.

When $\mu_{\mathrm{E}}$ is small enough that all of the phase factors lie between $-\pi$ and $\pi$, i.e.,

$$
\left|\frac{m}{n}+\frac{\mu_{\mathrm{E}}}{2 \pi}\right| \leq \frac{1}{2} \quad \text { for } m \in\left[-\frac{n-1}{2}, \frac{n-1}{2}\right],
$$

we will have $\ell_{m}=0$ for all $m$. If we assume the latter holds, the conformal dimension of the generalized twist operator $\tilde{\sigma}_{n}=\prod \tilde{\sigma}_{\alpha\left(m, \mu_{\mathrm{E}}\right)}$ becomes

$$
\Delta_{n}=\frac{1}{24}\left(n-\frac{1}{n}\right)+\frac{n}{2}\left(\frac{\mu_{\mathrm{E}}}{2 \pi}\right)^{2}
$$

and the charged Rényi entropy is given by

$$
\tilde{S}_{n}\left(\mu_{\mathrm{E}}\right)=\frac{1}{6}\left(1+\frac{1}{n}\right) \log |u-v| .
$$


That is, for small $\mu_{\mathrm{E}}$, the charged Rényi entropy is exactly the same as the result in eq. (2.63), i.e., the Rényi entropy without the Wilson line. As $\mu_{\mathrm{E}}$ increases, we can no longer choose all of the $\ell_{m}$ to be zero. The first transition occurs for $m=\frac{n-1}{2}$ when

$$
\frac{n-1}{2 n}+\frac{\mu_{\mathrm{E}}}{2 \pi}=\frac{1}{2} \longleftrightarrow \mu_{\mathrm{E}}=\frac{\pi}{n}
$$

beyond which the naive phase factor would be larger than $\pi$. Setting $\ell_{\frac{n-1}{2}}=-1$ and using the appropriate conformal dimension, we find that the charged Rényi entropy within the range $\frac{\pi}{n} \leq \mu_{\mathrm{E}}<\frac{3 \pi}{n}$ becomes

$$
\tilde{S}_{n}\left(\mu_{\mathrm{E}}\right)=\left[\frac{1}{6}\left(1+\frac{1}{n}\right)-\frac{4}{n-1}\left(\frac{\mu_{\mathrm{E}}}{2 \pi}-\frac{1}{2 n}\right)\right] \log |u-v| .
$$

Further phase transitions occur whenever $\mu_{\mathrm{E}}=\frac{\pi}{n}(2 k+1)$. For example, for $\frac{3 \pi}{n} \leq \mu_{\mathrm{E}}<$ $\frac{5 \pi}{n}$, the charged Rényi entropy becomes

$$
\tilde{S}_{n}\left(\mu_{\mathrm{E}}\right)=\left[\frac{1}{6}\left(1+\frac{1}{n}\right)+\frac{4}{n-1}\left(\frac{\mu_{\mathrm{E}}}{2 \pi}-\frac{5}{2 n}\right)\right] \log |u-v| .
$$

Of course, it is straightforward to extend these results to all values of $\mu_{\mathrm{E}}$. As can be anticipated from eq. (2.64), the charged Rényi entropy exhibits a periodicity

$$
\tilde{S}_{n}\left(\mu_{\mathrm{E}}\right)=\tilde{S}_{n}\left(\mu_{\mathrm{E}}+2 \pi\right) .
$$

Hence within a single period, there will be $n$ separate branches running from $\mu_{\mathrm{E}}=$ $\frac{\pi}{n}(2 k-1)$ to $\frac{\pi}{n}(2 k+1)$ for integer $k$. The result for $n=3$ is shown in figure 1 . Of course, these results show that the charged Rényi entropy is a non-analytic function of $\mu_{\mathrm{E}}$ and $n$. In particular, we might note that the apparent singularities at $n=1$ in eqs. (2.72) and (2.73) are not physical. As a final comment, we remark that the results derived here using twist operators agree with those coming from the heat kernel computations in Appendix A.

\section{Holographic computations}

In this section we calculate holographic Rényi entropies for boundary theories dual to Einstein gravity coupled to a Maxwell gauge field in the bulk. The relevant bulk solutions are charged topological black holes with hyperbolic horizons. These solutions represent the grand canonical ensemble of the boundary CFT on the hyperbolic cylinder. We only present the salient steps in the following calculations and refer the reader to [9] for a detailed description of how the holographic Rényi entropies are calculated. We consider boundary theories in dimension $d \geq 3$ here and provide holographic calculations for $d=2$ in appendix B. 


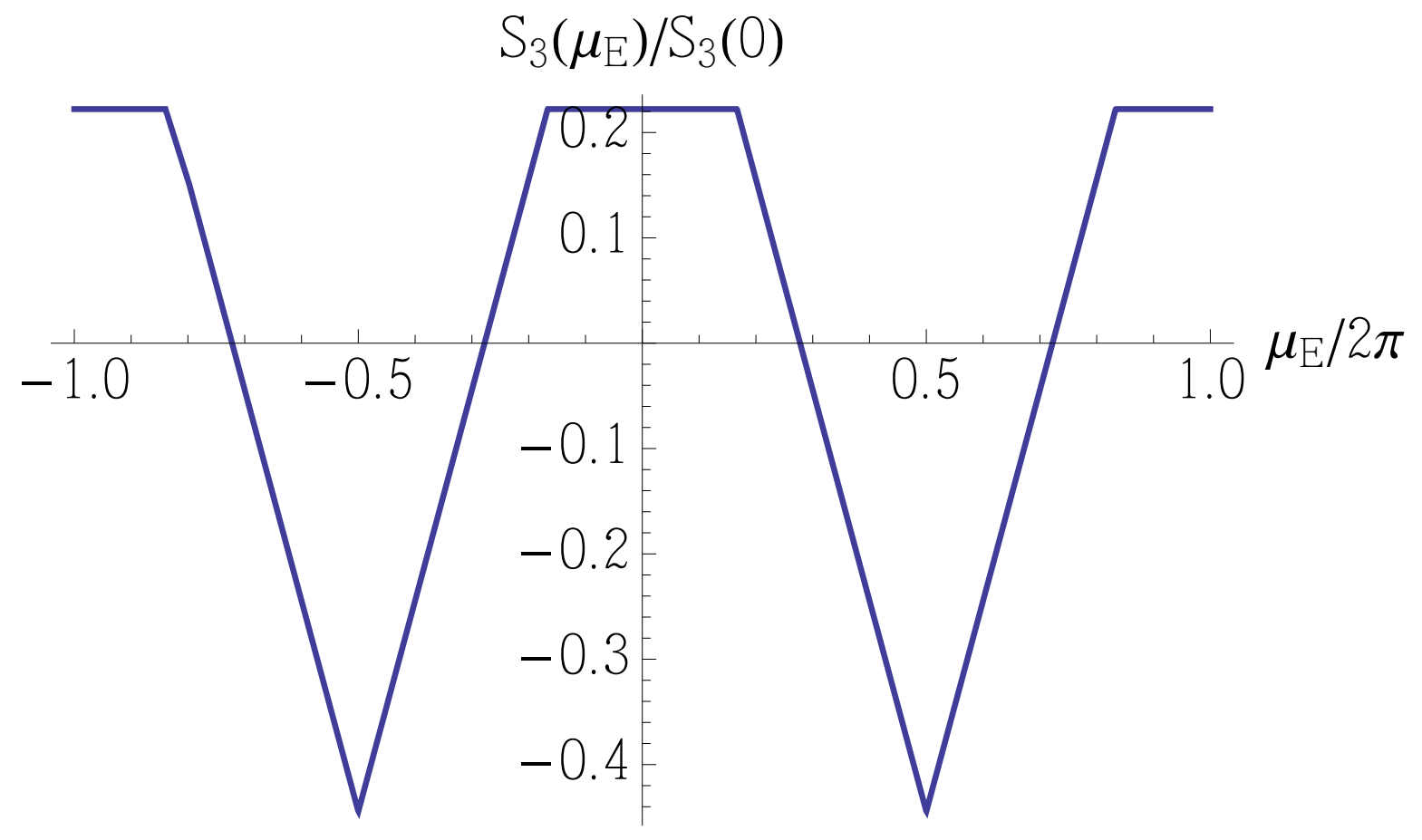

Figure 1: Charged Rényi entropy with $n=3$ for a two-dimensional free fermion as a function of $\mu_{\mathrm{E}}$.

\subsection{Charged black hole solution}

In $d+1$ bulk dimensions, we write the Einstein-Maxwell action with negative cosmological constant as ${ }^{13}$

$$
I_{E-M}=\frac{1}{2 \ell_{\mathrm{P}}^{d-1}} \int d^{d+1} x \sqrt{-g}\left(\frac{d(d-1)}{L^{2}}+\mathcal{R}-\frac{\ell_{*}^{2}}{4} F_{\mu \nu} F^{\mu \nu}\right) .
$$

For $d \geq 3$, the metric for the charged topological black hole takes the form

$$
d s^{2}=-f(r) \frac{L^{2}}{R^{2}} d \tau^{2}+\frac{d r^{2}}{f(r)}+r^{2} d \Sigma_{d-1}^{2},
$$

with

$$
f(r)=\frac{r^{2}}{L^{2}}-1-\frac{m}{r^{d-2}}+\frac{q^{2}}{r^{2 d-4}}
$$

where $d \Sigma_{d-1}^{2}=d u^{2}+\sinh ^{2} u d \Omega_{d-2}^{2}$ is the metric on $H^{d-1}$ with unit curvature. Note that the time coordinate is normalized here [8] so that the boundary metric naturally

\footnotetext{
${ }^{13}$ The scale $\ell_{*}$ appearing in the prefactor of the Maxwell term should be fixed by the details of the boundary theory. With this notation, the $(d+1)$-dimensional gauge coupling becomes $g_{5}^{2}=2 \ell_{\mathrm{P}}^{d-1} / \ell_{*}^{2}$.
} 
becomes $d s_{C F T}^{2}=-d \tau^{2}+R^{2} d \Sigma_{d-1}^{2}$, i.e., the Minkowski continuation of eq. (2.9). The corresponding bulk gauge field is

$$
A=\left(\sqrt{\frac{2(d-1)}{(d-2)}} \frac{L q}{R \ell_{*} r^{d-2}}-\frac{\mu}{2 \pi R}\right) d \tau,
$$

The chemical potential $\mu$ is fixed by requiring that the gauge field vanish at the horizon $r=r_{H}$, i.e.,

$$
\mu=2 \pi \sqrt{\frac{2(d-1)}{(d-2)}} \frac{L q}{\ell_{*} r_{H}^{d-2}} .
$$

The mass parameter $m$ is related to the horizon radius $r_{H}$ by

$$
m=\frac{r_{H}^{d-2}}{L^{2}}\left(r_{H}^{2}-L^{2}\right)+\frac{q^{2}}{r_{H}^{d-2}}
$$

Hence, we may rewrite the function $f(r)$ (3.3) in terms of the horizon radius $r_{H}$ and the charge $q$, giving

$$
f(r)=\frac{r^{2}}{L^{2}}-1+\frac{q^{2}}{r^{2 d-4}}-\left(\frac{r_{H}}{r}\right)^{d-2}\left(\frac{r_{H}^{2}}{L^{2}}-1+\frac{q^{2}}{r_{H}^{2 d-4}}\right) .
$$

The temperature of this black hole is given by

$$
T=\frac{T_{0}}{2} L f^{\prime}\left(r_{H}\right)=\frac{T_{0}}{2}\left[d \frac{r_{H}}{L}-(d-2) \frac{L}{r_{H}}\left(1+\frac{d-2}{2(d-1)}\left(\frac{\mu \ell_{*}}{2 \pi L}\right)^{2}\right)\right]
$$

where $T_{0}$ is the temperature given in eq. (2.10) and the 'prime' denotes differentiation with respect to $r$. The thermal entropy is given by the Bekenstein-Hawking formula

$$
S=\frac{2 \pi}{\ell_{\mathrm{P}}^{d-1}} V_{\Sigma} r_{H}^{d-1}
$$

where $V_{\Sigma}$ denotes the regulated (dimensionless) volume of the hyperbolic plane $H^{d-1}$, as described in [9]. Recall that this volume is a function of $R / \delta$, the ratio of the radius of the entangling sphere to the short-distance cut-off in the boundary theory. Further the leading contribution takes the form

$$
V_{\Sigma} \simeq \frac{\Omega_{d-2}}{d-2} \frac{R^{d-2}}{\delta^{d-2}}+\cdots
$$

where $\Omega_{d-2}=2 \pi^{(d-1) / 2} / \Gamma((d-1) / 2)$ is the area of a unit $(d-2)$-sphere. Hence the corresponding Rényi entropies in the following begin with an area law contribution. 
As a final comment, we note that we have presented the Minkowski-signature solution here with a real chemical potential $\mu$. This gives the holographic representation of the grand canonical ensemble on the hyperbolic cylinder $R \times H^{d-1}$. One can easily transform to Euclidean signature by replacing $\tau=-i \tau_{\mathrm{E}}$ to produce the dual of the thermal ensemble for the boundary CFT on $S^{1} \times H^{d-1}$. In this replacement, the form of the metric function $f(r)$ is unchanged and as usual, the Euclidean time is made periodic with $\Delta \tau_{\mathrm{E}}=1 / T$ to ensure that the bulk geometry is smooth at $r=r_{H}$. The gauge field becomes imaginary for this Euclidean bulk solution. Of course, the latter

is in keeping with our discussion of the Euclidean path integral in section 2, where an imaginary background gauge field was introduced to describe the grand canonical ensemble. Here, this background field in the boundary theory is simply given by the non-normalizable of the bulk gauge field, i.e., $B_{\mu}=-\lim _{r \rightarrow \infty} A_{\mu}$.

\subsection{Charged Rényi entropies}

Applying eq. (2.19) with a real chemical potential, we see that the charged Rényi entropy for a spherical entangling surface can be expressed as

$$
S_{n}(\mu)=\frac{n}{n-1} \frac{1}{T_{0}} \int_{x_{n}}^{x_{1}} S(x, \mu) \partial_{x} T(x, \mu) d x
$$

where $x=r_{H} / L$ and $S(x, \mu)$ is the horizon entropy (3.9). Evaluating eq. (3.8) in terms of $x$ gives

$$
T(x, \mu)=\frac{T_{0}}{2 x}\left(d x^{2}-(d-2)-\frac{(d-2)^{2}}{2(d-1)}\left(\frac{\mu \ell_{*}}{2 \pi L}\right)^{2}\right) .
$$

Then $x_{n}$ is the largest solution of $T\left(x_{n}, \mu\right)=T_{0} / n$ and is given by

$$
x_{n}=\frac{1}{d n}+\sqrt{\frac{1}{d^{2} n^{2}}+\frac{d-2}{d}+\frac{(d-2)^{2}}{2 d(d-1)}\left(\frac{\mu \ell_{*}}{2 \pi L}\right)^{2}} .
$$

Combining these expressions then yields

$$
S_{n}(\mu)=\pi V_{\Sigma}\left(\frac{L}{\ell_{\mathrm{P}}}\right)^{d-1} \frac{n}{n-1}\left[\left(1+\frac{d-2}{2(d-1)}\left(\frac{\mu \ell_{*}}{2 \pi L}\right)^{2}\right)\left(x_{1}^{d-2}-x_{n}^{d-2}\right)+x_{1}^{d}-x_{n}^{d}\right] .
$$

Note that when $\ell_{*}=0$, eqs. (3.13) and (3.14) reduce to the results found in [9]. 
Expressions for the charged Rényi entropy with specific choices for $n$ are:

$$
\begin{aligned}
\lim _{n \rightarrow 0} S_{n} & =\pi V_{\Sigma}\left(\frac{L}{\ell_{\mathrm{P}}}\right)^{d-1}\left(\frac{2}{d}\right)^{d} \frac{1}{n^{d-1}} \\
S_{\mathrm{EE}}=\lim _{n \rightarrow 1} S_{n} & =\pi V_{\Sigma}\left(\frac{L}{\ell_{\mathrm{P}}}\right)^{d-1} \frac{(d-2) x_{1}^{d-2}}{d x_{1}-1}\left(1+\frac{d-2}{2(d-1)}\left(\frac{\mu \ell_{*}}{2 \pi L}\right)^{2}+\frac{d x_{1}^{2}}{d-2}\right) \\
S_{2} & =2 \pi V_{\Sigma}\left(\frac{L}{\ell_{\mathrm{P}}}\right)^{d-1}\left(\left(1+\frac{d-2}{2(d-1)}\left(\frac{\mu \ell_{*}}{2 \pi L}\right)^{2}\right)\left(x_{1}^{d-2}-x_{2}^{d-2}\right)+x_{1}^{d}-x_{2}^{d}\right) \\
\lim _{n \rightarrow \infty} S_{n} & =\pi V_{\Sigma}\left(\frac{L}{\ell_{\mathrm{P}}}\right)^{d-1}\left(\left(1+\frac{d-2}{2(d-1)}\left(\frac{\mu \ell_{*}}{2 \pi L}\right)^{2}\right)\left(x_{1}^{d-2}-x_{\infty}^{d-2}\right)+x_{1}^{d}-x_{\infty}^{d}\right)
\end{aligned}
$$

where

$$
x_{\infty}^{2}=\frac{d-2}{d}\left(1+\frac{d-2}{2(d-1)}\left(\frac{\mu \ell_{*}}{2 \pi L}\right)^{2}\right) .
$$

Another interesting limit to consider is holding $n$ fixed while $\mu \rightarrow \infty$, which yields

$$
\lim _{\mu \rightarrow \infty} S_{n}(\mu)=2 \pi V_{\Sigma}\left(\frac{(d-2)^{2}}{2 d(d-1)}\right)^{\frac{d-1}{2}}\left(\frac{\ell_{*}}{\ell_{\mathrm{P}}}\right)^{d-1}\left(\frac{\mu}{2 \pi}\right)^{d-1} .
$$

Hence we have the curious result that, to leading order in $\mu$, the Rényi entropies are independent of $n$ in this limit.

(a)

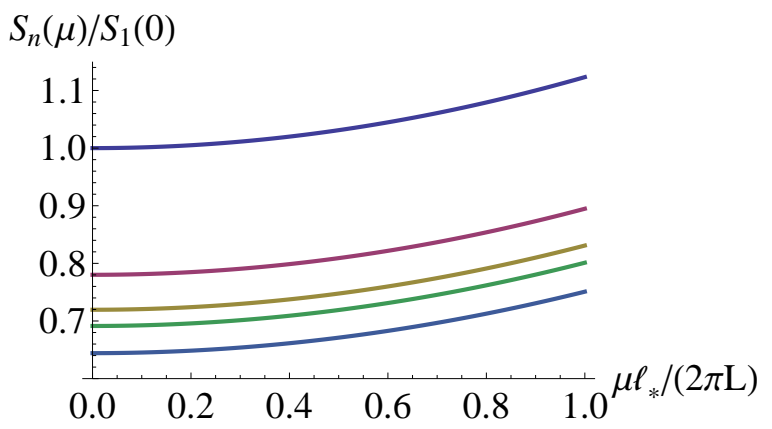

(b)

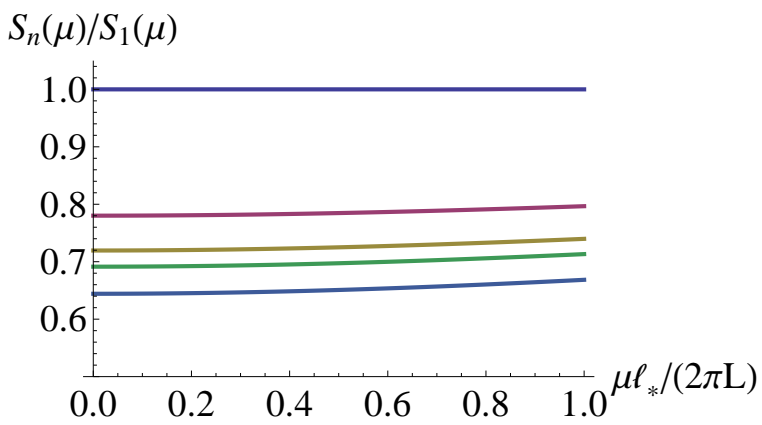

Figure 2: The $d=3$ charged Rényi entropy (normalized by (a) $S_{1}(0)$ and (b) $S_{1}(\mu)$ ) as a function of $\mu$. The curves correspond to (from top to bottom) $n=1,2,3,4,10$

The results for the charge Rényi entropy (3.14) are illustrated in figures 2 and 3, which plot $S_{n}(\mu)$ as a function of $\mu$ for various values of $n$ in $d=3$ and 4 . In these figures, it is evident that for fixed $\mu$, the Rényi entropy decreases as $n$ increases. This 
(a)

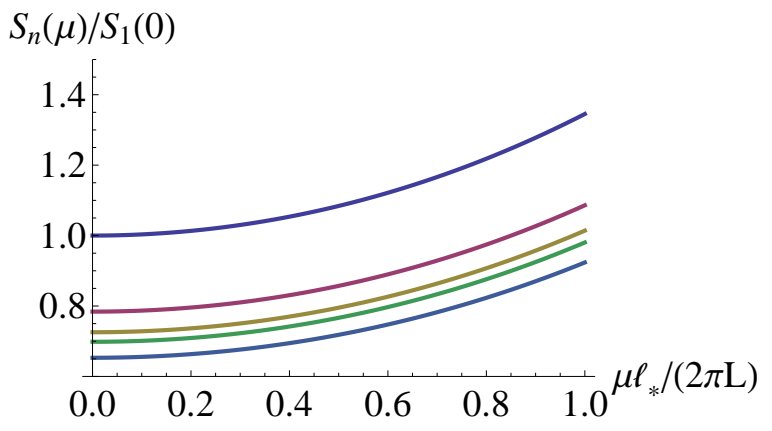

(b)

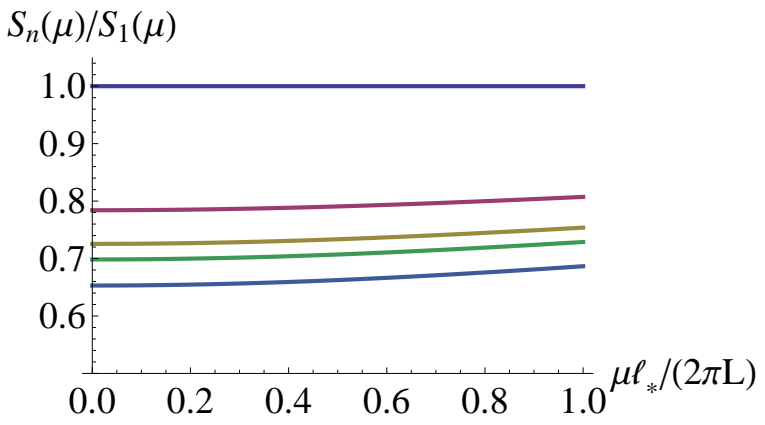

Figure 3: The $d=4$ charged Rényi entropy (normalized by (a) $S_{1}(0)$ and (b) $S_{1}(\mu)$ ) as a function of $\mu$. The curves correspond to (from top to bottom) $n=1,2,3,4,10$

behaviour is also shown in figure 4 a where the charged Rényi entropy in $d=3$ is shown as a function of $n$. The figure shows very clearly in this example that $\partial_{n} S_{n}(\mu)<0$. As discussed in [9] (see also [7]), standard Rényi entropies must satisfy various inequalities:

$$
\begin{aligned}
\frac{\partial S_{n}}{\partial n} & \leq 0, & \frac{\partial}{\partial n}\left(\frac{n-1}{n} S_{n}\right) & \geq 0, \\
\frac{\partial}{\partial n}\left((n-1) S_{n}\right) & \geq 0, & \frac{\partial^{2}}{\partial n^{2}}\left((n-1) S_{n}\right) & \leq 0 .
\end{aligned}
$$

By examining plots of the numerical results, e.g., see $\frac{n-1}{n} S_{n}(\mu)$ in figure $4 \mathrm{~b}$, we find that these inequalities still appear to hold in the charged case. The analysis in [9] found that this result essentially follows from the connection between the Rényi entropies for a spherical entangling surface and the thermal entropy on the hyperbolic cylinder $R \times H^{d-1}$. In particular, it follows that these inequalities (3.18) will be satisfied for any $\mathrm{CFT}$, as long as the corresponding thermal ensemble is stable. Thus we expect that the inequalities (3.18) will continue to hold for charged Rényi entropies. Of course, the arguments in [9] will not apply where the Rényi entropies exhibit phase transitions [26], as discussed in section 3.4.

As we mentioned above, both real and imaginary chemical potentials are of interest. Our holographic results are easily analytically continued to imaginary chemical potential by simply replacing $\mu=i \mu_{\mathrm{E}}$ and $q=i q_{\mathrm{E}}$. Note that with this replacement, the root $x_{n}$ in eq. (3.13) fails to exist if $\mu_{\mathrm{E}}$ becomes too large. The region of validity of analytically continued solutions is given by

$$
\mu_{\mathrm{E}}^{2} \leq \frac{8 \pi^{2}(d-1)}{d-2}\left(\frac{L}{\ell_{*}}\right)^{2}\left(1+\frac{1}{d(d-2) n^{2}}\right) .
$$


(a)

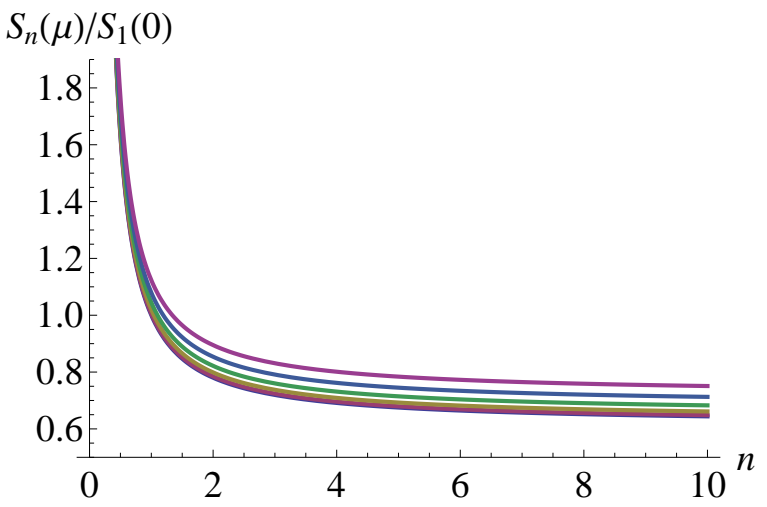

(b)

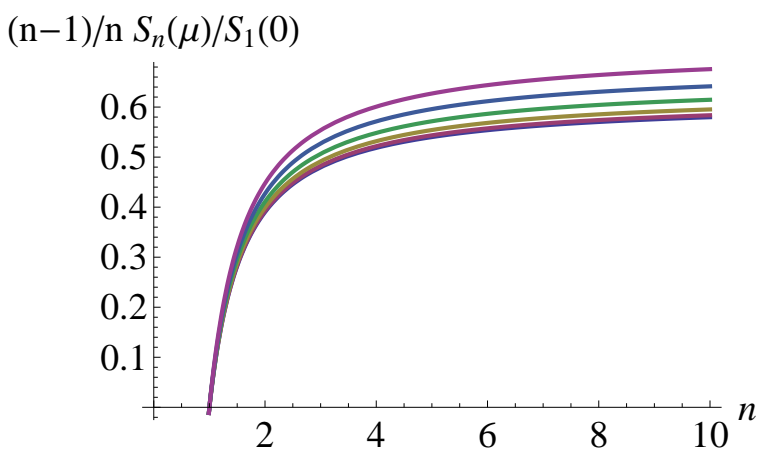

Figure 4: The charged Rényi entropy (normalized by $\left.S_{1}(0)\right)$ in $d=3$ shown function of $n$ in panel (a). In panel (b), we show $\frac{n-1}{n} S_{n}(\mu)$ as a function of $n$. Note that the slope of the curves is negative in panel (a) and positive in panel (b). In both cases, the curves correspond to (from top to bottom) $\frac{\mu \ell_{*}}{2 \pi L}=1.0,0.8,0.6,0.4,0.2$ and 0.0 .

If $\mu_{\mathrm{E}}$ increases beyond this bound (with fixed $n$ ), the event horizon disappears and we are left with a naked singularity. Typical results for the charged Rényi entropy with imaginary chemical potential are shown in figure 5. In comparing the figures, we see that while the charged Rényi entropy increases slowly with increasing $\mu$ in figures 2 and $3, \tilde{S}_{n}\left(\mu_{\mathrm{E}}\right)$ decreases, and in a much more dramatic fashion, as $\mu_{\mathrm{E}}$ increases in figure 5.

(a)

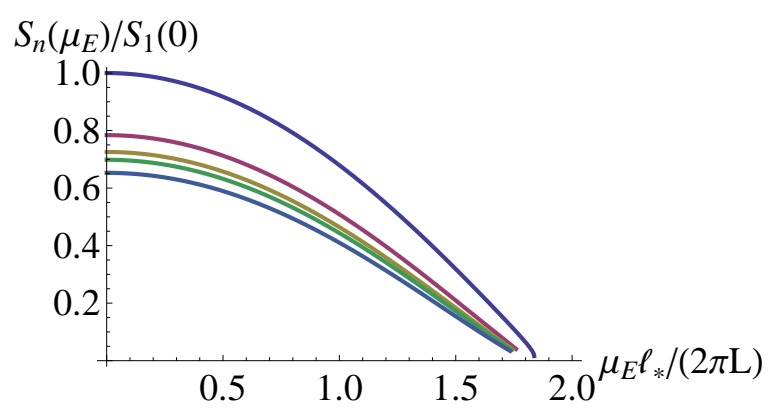

(b)

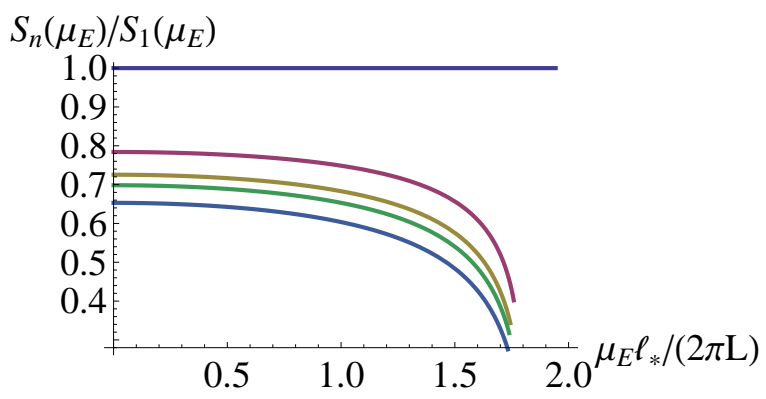

Figure 5: The $d=4$ charged Rényi entropy (normalized by (a) $\tilde{S}_{1}(0)$ and (b) $\tilde{S}_{1}\left(\mu_{\mathrm{E}}\right)$ ) as a function of the imaginary chemical potential $\mu_{\mathrm{E}}$. The curves correspond to (from top to bottom) $n=1,2,3,4,10$ 


\subsection{Twist operators}

In section 2.3, we derived various expressions for the conformal weight and the magnetic response of the twist operators, as well as various expansion coefficients appearing in these. Here we wish to examine these properties of the twist operators in the boundary CFT dual to the Einstein-Maxwell theory (3.1).

To begin, recall eq. (2.21) for the conformal weight of the twist operator,

$$
h_{n}(\mu)=\frac{2 \pi n}{d-1} R^{d}\left(\mathcal{E}\left(T_{0}, \mu=0\right)-\mathcal{E}\left(T_{0} / n, \mu\right)\right) .
$$

The details of the holographic calculation of the corresponding energy densities are given in appendix C.1. Then using eq. (C.4), the desired conformal weight can be written as

$$
h_{n}(\mu)=n \pi \frac{L^{d-1}}{\ell_{\mathrm{P}}^{d-1}}\left(x_{n}^{d-2}\left(1-x_{n}^{2}\right)-\frac{x_{n}^{d-2}(d-2)}{2(d-1)}\left(\frac{\mu \ell_{*}}{2 \pi L}\right)^{2}\right) .
$$

Here we might note that this result can be expressed entirely in terms of parameters in the boundary theory using eqs. (C.15) and (C.18), which show that $L^{d-1} / \ell_{\mathrm{P}}^{d-1} \sim C_{T}$ and $\ell_{*}^{2} L^{d-3} / \ell_{\mathrm{P}}^{d-1} \sim C_{V}$.

Next we would like to recover eqs. (2.26) and (2.45) for the expansion coefficients of the above conformal weight. Hence given eq. (3.21), we evaluated the following two coefficients:

$$
\begin{aligned}
& h_{10}=\left.\partial_{n} h_{n}(\mu)\right|_{n=1, \mu=0}=\frac{2 \pi}{d-1} \frac{L^{d-1}}{\ell_{\mathrm{P}}^{d-1}}, \\
& h_{02}=\left.\partial_{\mu}^{2} h_{n}(\mu)\right|_{n=1, \mu=0}=-\frac{(d-2)(2 d-3)}{4 \pi(d-1)^{2}} \frac{\ell_{*}^{2} L^{d-3}}{\ell_{\mathrm{P}}^{d-1}} .
\end{aligned}
$$

At this point, using eq. (C.18), we can substitute for $C_{T}$ in terms of the ratio $L^{d-1} / \ell_{\mathrm{P}}^{d-1}$ in eq. (2.26) and we recover precisely eq. (3.22). Similarly, using eqs. (C.16) and (C.17) in eq. (2.45), we reproduce exactly the expression in eq. (3.23). We also note that $h_{01}=\left.\partial_{\mu} h_{n}(\mu)\right|_{n=1, \mu=0}=0$ from eq. (3.21), which is in agreement with our general expectations in section 2.3 .

Now we turn our attention to the magnetic response, which was given by eq. (2.25),

$$
k_{n}(\mu)=2 \pi n R^{d-1} \rho(n, \mu) .
$$

Now in the holographic framework, we know that the charge density, i.e., $\left\langle J_{\tau}\right\rangle$ is determined by the normalizable component of the gauge field (3.4), however, we leave 
its determination, including the precise normalization, to appendix C.2. Substituting the holographic result (C.8) into eq. (3.24), we find the magnetic response in our holographic model is

$$
k_{n}(\mu)=\frac{d-2}{2} \frac{\ell_{*}^{2} L^{d-3}}{\ell_{\mathrm{P}}^{d-1}} n x_{n}^{d-2} \mu .
$$

Again, this result can be expressed entirely in terms of parameters of the boundary CFT using eq. (C.15). As above, we would like to examine the expansion coefficients of the above magnetic response and hence we calculate

$$
\begin{gathered}
k_{01}=\left.\partial_{\mu} k_{n}(\mu)\right|_{n=1, \mu=0}=\frac{d-2}{2} \frac{\ell_{*}^{2} L^{d-3}}{\ell_{\mathrm{P}}^{d-1}}, \\
k_{11}=\left.\partial_{n} \partial_{\mu} k_{n}(\mu)\right|_{n=1, \mu=0}=\frac{d-2}{2(d-1)} \frac{\ell_{*}^{2} L^{d-3}}{\ell_{\mathrm{P}}^{d-1}} .
\end{gathered}
$$

These two coefficients can then be compared with eqs. (2.55) and (2.56) using the results in appendix C.3. As expected, the holographic expression (C.15) of $C_{V}$ yields a precise agreement of eq. (3.26) above with eqs. (2.55) and (2.57) in section 2.3. Similarly, eqs. (2.56) and (3.27) match exactly using eqs. (C.16) and (C.17).

\subsection{Thermodynamics, stability and phase transitions}

It is natural to investigate the thermodynamical properties of the charged hyperbolic black holes. Thermodynamical instability in some regions of phase space could be responsible for interesting features in the Rényi entropies. One could also imagine that at low temperatures, a different geometry would be prefered over the black hole phase, and the system would go through a Hawking-Page phase transition as the temperature is increased. It turns out none of these features occur for charged hyperbolic black hole in the grand canonical ensemble. The Gibbs free energy $G=\left(M-M_{c}\right)-T S-\mu q$ was calculated in [27]:

$$
G=-\frac{V_{\Sigma} r_{H}^{d-2}}{2 \ell_{\mathrm{P}}^{d-1}}\left(1+\frac{(d-2)}{2(d-1)}\left(\frac{\mu \ell_{*}}{2 \pi L}\right)^{2}+\frac{r_{H}^{2}}{L^{2}}-m_{c}(\mu)\right)
$$

where $m_{c}(\mu)$ is the critical mass at which the temperature vanishes, i.e.,

$$
m_{c}=-2(d-1) r_{c}^{n-1}\left(1-\frac{(d-1) r_{c}^{2}}{(d-2) L^{2}}\right) \quad \text { with } \quad \frac{r_{c}^{2}}{L^{2}}=\frac{d-2}{d}\left(1+\frac{(d-2)}{2(d-1)}\left(\frac{\mu \ell_{*}}{2 \pi L}\right)^{2}\right)
$$

One can check that the Gibbs energy is always negative and equals zero when the black hole is extremal, excluding any Hawking-Page phase transition. The specific heat was 
calculated in [27] as well:

$$
C_{\mu}=T\left(\frac{\partial S}{\partial T}\right)_{\mu}=\frac{8 \pi^{2}(d-1) V_{\Sigma} T r_{H}^{d}}{(d-2) \ell_{\mathrm{P}}^{d-1}}\left(1+\frac{d}{d-2} \frac{r_{H}^{2}}{L^{2}}+\frac{d-2}{d-1}\left(\frac{\mu \ell_{*}}{2 \pi L}\right)^{2}\right)^{-1}
$$

which is always positive, meaning the black holes are thermodynamically stable.

Before leaving the subject of instabilities, it is interesting to note that the presence of a light scalar in the bulk would render the black holes unstable at low temperatures. Indeed, the extremal charged black hole has a $A d S_{2} \times H^{d-1}$ near horizon geometry, where the relative radii of the two spaces depends on the charge:

$$
L_{A d S_{2}}^{2}=\frac{2 L_{A d S_{d+1}}^{2}}{f^{\prime \prime}\left(r_{H}\right)} \quad L_{H^{d-1}}^{2}=r_{H}^{2}
$$

with $f(r)$ is the metric function in eq. (3.3) above. For simplicity, let us consider a neutral scalar. The extremal black hole will develop scalar hair if the mass of the scalar is below the BF bound of the near-horizon $A d S_{2}$. We wish to consider normalizable modes and these must depend on the hyperbolic coordinates, as the volume of the hyperboloid is infinite. Normalizable modes can be expanded in eigenvalues of the Laplacian as $\nabla_{H^{d-1}}^{2} \phi=-\lambda \phi$ with $\lambda>(d-2)^{2} / 4$ and near the horizon, this Laplacian will generate an effective shift of the mass of the scalar. Thus we exepct an instability if the scalar mass $M$ lies in the range

$$
-\frac{d^{2}}{4}<M^{2} L^{2}<-\frac{f^{\prime \prime}\left(r_{H}\right)}{8}-\frac{(d-2)^{2}}{4 r_{H}^{2}} .
$$

It turns out the two terms on the right-hand side of this equation combine in such a way that the answer does not depend on charge:

$$
-\frac{d^{2}}{4}<M^{2} L^{2}<-\frac{d(d-1)}{4}
$$

found in [26]. It seems that at zero temperature, a neutral scalar will not detect changes in the geometry induced by charge. Turning our attention to charged scalars, it was noted in [28] that the effect of the charge will be to induce a shift in the scalar mass. This only makes things worse and a scalar instability is therefore expected as well. We leave the detailed analysis of these effects for future work but note that the Rényi entropies should exhibit phase transitions as $n$ is varied, if light scalars are present in the bulk spectrum [29]. 


\section{Discussion}

We have examined a new class of entanglement measures (1.2) which extend the usual definition of Rényi entropy to include a chemical potential for a conserved global charge. These charged Rényi entropies measure the degree of entanglement in different charge sectors of the theory. As described in section 2, the evaluation of these entropies proceeds as usual with a Euclidean path integral, but with the addition of a (fixed) background gauge field which introduces a Wilson line, proportional to the chemical potential, around the entangling surface. The latter can be interpreted as binding a sheet of magnetic flux to the standard twist operators which appear in evaluating the Rényi entropy.

For the special case of a CFT with a spherical entangling surface, we can apply a conformal transformation to map charged Rényi entropies to the thermal entropies of a grand canonical ensemble, albeit on the hyperbolic cylinder $R \times H^{d-1}$. This allows us to study the properties of the generalized twist operators, as discussed in section 2.3. In particular, the conformal weight and the magnetic response of these twist operators are related to the energy density and the charge density, respectively, in the thermal ensemble on the hyperbolic cylinder. These two parameters are functions of both $n$ and $\mu$ and exhibit certain universal characteristics when expanded in the vicinity of $n=1$ and $\mu=0$.

In section 3, we considered computations of charged Rényi entropies using holography, where they are related to the thermal entropy of charged black holes with hyperbolic horizons. In addition to determining the charged Rényi entropy, we were able to determine the conformal weight and magnetic response of the corresponding twist operators in this holographic model. In a particular, we were able to recover the universal behaviour exhibited by the expansion coefficients in, e.g., eq. (2.57). In section 2.4 and appendix A, we described the computation of charged Rényi entropies for free field theories. We found the results to be in qualitative agreement with our holographic calculations. A particularly interesting point of comparison is $d=2$, which was considered in section 2.4 for free fermions, and in appendix B for holographic models. For free bosons, we observed that the Rényi entropy is non-analytic at $\mu_{\mathrm{E}}=0$. Thus, while there is a range where free fermions can be analytically continued to the real chemical potential, free bosons can not be so continued.

We found that the charged Rényi entropy in the holographic model obeyed various inequalities (3.18), which were originally established for the standard Rényi entropy without a chemical potential. Following [9], we argued that the stability of the grand canonical ensemble on the hyperbolic geometry was sufficient to guarantee these inequalities would be satisfied by the charged Rényi entropy. However, if one examines 
the origin of these inequalities [7], the derivation only relied on the fact that the Rényi entropies are moments of a probability distribution with $p_{i}>0$ and $\sum_{i} p_{i}=1$. The same statement applies for the charged Rényi entropies (with real chemical potential) and so we can expect that eq. (3.18) will be satisfied quite broadly for these new entanglement measures. It would be interesting to explicitly study the validity of these inequalities for more general QFT's and choices of entangling surface. At the same time, it would be interesting to investigate whether derivatives of $S_{n}(\mu)$ with respect to $\mu$ also satisfy any general properties. For example, in figures (2) and (3), it seems that

$\mu \frac{\partial S_{n}(\mu)}{\partial \mu} \geq 0$ for our holographic model. Note that an imaginary chemical potential does not respect the above inequality. In particular, the Rényi entries and the free energies can take negative values. The analytic continuation between the imaginary and the real chemical potentials is non-trivial because of poles and branch cuts. In gravity, the regular black hole space time ceases to exist for large $\mu_{\mathrm{E}}$, i.e., eq. (3.19).

There are several natural generalizations of the investigations presented here. For example, the holographic computations could be extended to consider bulk theories with higher derivative interactions (e.g., Gauss-Bonnet or $F^{4}$ terms), following [30-32]. Another interesting direction would be to connect our holographic calculations to the large- $N$ limit of super Rényi entropy for the ABJM model in [14].

It may also be of interest to consider a generalization of the Rényi entropy for fixed charge ensembles (instead of fixing the chemical potential). Here, the holographic computations may produce some interesting new behaviour. Finally, in the case of a spherical entangling surface (where the system is rotationally invariant) it is natural to label the states by their angular momentum and introduce a conjugate chemical potential to produce a 'rotating Rényi entropy' — see also [11]. The corresponding holographic calculations would involve more general classes of spinning hyperbolic black holes. A study of such rotating Rényi entropies at fixed angular potential, as well as charge, could follow very closely the present discussion. Results along these lines will be presented in [33].

\section{Acknowledgments}

We would like to thank Horacio Casini, Steve Shenker and especially Tadashi Takayanagi for helpful discussions. Research at Perimeter Institute is supported by the Government of Canada through Industry Canada and by the Province of Ontario through the Ministry of Research \& Innovation. AM and RCM gratefully acknowledge support from NSERC Discovery grants. Research by RCM is further supported by funding from the Canadian Institute for Advanced Research. TS acknowledges support from an NSERC Graduate Fellowship. 


\section{A Free QFT computations}

Here we consider various calculations of charged Rényi entropies for free fields using the heat kernel methods on hyperbolic spaces, and also by direct summing of appropriate modes on spheres. These QFT computations are most readily done if the chemical potential takes is purely imaginary values, i.e., $\mu=i \mu_{\mathrm{E}}$ where $\mu_{\mathrm{E}}$ is real. In this case, the chemical potential produces to a non-trivial boundary condition. As in section 2, we are interested in conformal theories and hence we consider calculations for a massless conformally coupled and complex scalar and for a free massless Dirac fermion. In both cases, the global charge is simply related with phase rotations of the corresponding field.

\section{A.1 Heat kernels on $S^{1} \times H^{d-1}$}

To begin, we gather a few useful results for heat kernel methods [34]. First, heat kernels on a product manifold factorize, for both fermions and bosons,

$$
K_{S^{1} \times H^{d-1}}\left(\left\{x_{i}\right\},\left\{y_{i}\right\}, t\right)=K_{S^{1}}\left(x_{1}, y_{1}, t\right) K_{H^{d-1}}\left(x_{2, \cdots, d}, y_{2, \cdots, d}, t\right)
$$

The total free energy in $S^{1} \times H^{d-1}$ is

$$
F=-\frac{(-)^{f}}{2} \int d^{d} x \frac{d t}{t} e^{-m_{c}(1-f) t} K_{S^{1} \times H^{d-1}}(x, x, t)
$$

where $f=1$ for spin half Dirac fermions, and $f=0$ for scalars. The conformal mass $m_{c}$ in $H^{d-1}$ for the conformally coupled scalar is

$$
m_{c}=-\frac{(d-2)^{2}}{4 R^{2}}
$$

where $R$ is the radius of $H^{d-1}$. For convenience, we will set $R=1$ in the following.

We will consider finite temperature and purely imaginary chemical potential $\mu_{E}=$ $i \mu_{\mathrm{E}}$ for a $U(1)$ global symmetry. This can be incorporated into the heat kernel by setting appropriate boundary conditions. For example, with inverse temperature $\beta=2 \pi n$ we have the boundary condition

$$
K_{S^{1} \times H^{d-1}}\left(\left\{x_{1}+2 \pi n, \cdots, x_{d}\right\},\left\{y_{1}, \cdots, y_{d}\right\}, t\right)=(-)^{f} e^{i n \mu_{\mathrm{E}}} K_{S^{1} \times H^{d-1}}(\{x\},\{y\}, t)
$$

Let us first focus on $K_{S^{1}}\left(x_{1}, y_{1}, t\right)$. It is not hard to show that

$$
K_{\mathbb{R}^{1}}\left(x_{1}, y_{1}, t\right)=\frac{1}{\sqrt{4 \pi t}} e^{-\frac{\left(x_{1}-y_{1}\right)^{2}}{4 t}} .
$$


Summing over images we find

$$
K_{S^{1}}\left(x_{1}, y_{1}, t\right)=\frac{1}{\sqrt{4 \pi t}} \sum_{m \in \mathbb{Z}} e^{\frac{-\left(y_{1}-x_{1}+2 \pi m\right)^{2}}{4 t}} e^{\left(-i n \mu_{\mathrm{E}}-i \pi f\right) m},
$$

which satisfies the boundary condition A.5. Note that using this method, $n \mu_{\mathrm{E}}<1$. The final result for $\mu_{\mathrm{E}} n>1$ should be obtained from $\mu_{\mathrm{E}} n<1$ by folding. We therefore expect discontinuities in the free energies when $\mu_{\mathrm{E}} n$ takes integer values.

In the case where $\mu_{\mathrm{E}}=0$ we recover the usual heat kernel at finite temperature $\beta=2 \pi n$. The $S^{1}$ circle has radius $2 \pi n$.

The heat kernel for massless scalars in $H^{D}$ takes the form

$$
K_{H^{2 x+1}}^{b}(\rho, t)=\frac{1}{(4 \pi t)^{1 / 2}}\left(\frac{-1}{2 \pi R^{2} \sinh \rho} \frac{\partial}{\partial \rho}\right)^{n} e^{-x^{2} t / R^{2}-\rho^{2} R^{2} /(4 t)},
$$

for hyperbolic spaces of odd dimensions, and

$$
K_{H^{2(x+1)}}^{b}(\rho, t)=e^{-(2 x+1)^{2} t /\left(4 R^{2}\right)}\left(\frac{-1}{2 \pi R^{2} \sinh \rho} \frac{\partial}{\partial \rho}\right)^{n} f_{H^{2}}(\rho, t),
$$

where $x$ is an integer, and $\rho$ is the geodesic distance between two points $x$ and $y$. The function $f_{H^{2}}(\rho, t)$ is defined as

$$
f_{H^{2}}(\rho, t)=\frac{\sqrt{2}}{(4 \pi t)^{3 / 2}} \int_{\rho}^{\infty} d \tilde{\rho} \frac{\tilde{\rho} e^{-\tilde{\rho}^{2} /(4 t)}}{\sqrt{\cosh \tilde{\rho}-\cosh \rho}}
$$

For fermions, we have

$$
K_{H^{2 x+1}}^{f}(\rho, t)=U(x, y) \cosh (\rho / 2)\left(\frac{-1}{2 \pi} \frac{\partial}{\partial \cosh \rho}\right)^{n} \cosh (\rho / 2)^{-1} \frac{e^{-\rho^{2} /(4 t)}}{\sqrt{4 \pi t}}
$$

and

$$
K_{H^{2 x}}^{f}(\rho, t)=U(x, y) \cosh (\rho / 2)\left(\frac{-1}{2 \pi} \frac{\partial}{\partial \cosh \rho}\right)^{n} \cosh (\rho / 2)^{-1} k_{H^{2}}(\rho, t)
$$

and

$$
K_{H^{2}}(\rho, t)=\frac{\sqrt{2} \cosh ^{-1}(\rho / 2)}{(4 \pi t)^{3 / 2}} \int_{\rho}^{\infty} d \tilde{\rho} \frac{\tilde{\rho} \cosh \tilde{\rho} / 2 e^{-\tilde{\rho}^{2} /(4 t)}}{\sqrt{\cosh \tilde{\rho}-\cosh \rho}}
$$

The matrix $U(x, y)$ has a trace given by $2^{[d / 2]}$, where $[\cdots]$ denotes the integer part. It counts the dimension of spinor space in $d$ dimensions.

From eq. (2.4), we can write the charged Rényi entropy as

$$
S_{n}=\frac{F\left(n, \mu_{\mathrm{E}}\right)-n F\left(1, \mu_{\mathrm{E}}\right)}{n-1}
$$

where $F\left(n, \mu_{\mathrm{E}}\right) \equiv-\log Z_{n}\left(\mu_{\mathrm{E}}\right)$ is the free energy evaluated at temperature $\beta=2 \pi n$ and chemical potential $\mu_{\mathrm{E}}$. We are thus ready to compute free energies at different dimensions. 


\section{A.1.1 $d=2$}

At temperature $\beta_{n}=2 \pi n$ and finite chemical potential $\mu_{\mathrm{E}}$, the free energy is

$$
F\left(n, \mu_{\mathrm{E}}\right)=\frac{(-)^{f}}{2}(2 \pi n) V_{H^{d-1}} \int \frac{d t}{t} \sum_{m} \frac{e^{-\pi^{2} m^{2} / t}}{\sqrt{4 \pi t}} e^{\left(-i n \mu_{\mathrm{E}}-i \pi f\right) m} K_{H^{1}}(\rho=0, t) .
$$

The heat kernel is

$$
K_{H^{1}}(0, t)=K_{\mathbb{R}}(0, t)=\frac{1}{\sqrt{4 \pi t}} .
$$

The free energy $F\left(n, \mu_{\mathrm{E}}\right)$ is divergent due to the $m=0$ mode in the $S^{1}$ heat kernel. For $m=0$ the contribution is linear in $n$, where $n$ appears as the overall volume factor from $S^{1}$. This dependence therefore drops out from $S_{n}$. We could therefore rewrite the regulated free energy $\hat{F}\left(n, \mu_{\mathrm{E}}\right)$ as

$$
\begin{aligned}
\hat{F}\left(n, \mu_{\mathrm{E}}\right) & =\frac{(-)^{f}}{2}(2 \pi n) V_{H^{d-1}} \int \frac{d t}{t} \sum_{m \in \mathbb{Z}_{+}} \frac{e^{-\pi^{2} m^{2} / t}}{\sqrt{4 \pi t}} 2 \cos \left(\left(n \mu_{\mathrm{E}}+\pi f\right) m\right) K_{H^{1}}(0, t) \\
& =\frac{(-)^{f}}{2} V_{H^{d-1}} \sum_{m \in \mathbb{Z}_{+}} \frac{8 \cos \left(n m \mu_{\mathrm{E}}\right) \cos (m \pi f)}{8 \pi^{2} n m^{2}} \\
& =\frac{(-)^{f}}{4 \pi^{2} n} V_{H^{d-1}}\left(\operatorname{Li}_{2}\left(e^{i n \mu_{\mathrm{E}}+i \pi f}\right)+\operatorname{Li}_{2}\left(e^{-i n \mu_{\mathrm{E}}-i \pi f}\right)\right)
\end{aligned}
$$

For $0 \leq \operatorname{Re}(x)<1$ and $\operatorname{Im}(x) \geq 0$, or $\operatorname{Re}(x) \geq 1$ and $\operatorname{Im}(x)<0$

$$
\mathrm{Li}_{2}\left(e^{2 \pi i x}\right)+\mathrm{Li}_{2}\left(e^{-2 \pi i x}\right)=-\frac{(2 \pi i)^{2}}{2} B_{2}(x)=-\frac{(2 \pi)^{2}}{\Gamma[2]} \zeta(-1, x),
$$

where $B_{2}$ is the Bernoulli polynomial, and $\zeta(a, b)$ the Hurwitz zeta function. We are left with

$\hat{F}\left(n, \mu_{\mathrm{E}}\right)=\frac{(-)^{f} V_{H^{1}}}{2 n} B_{2}\left(\frac{n \mu_{\mathrm{E}}}{2 \pi}+\frac{f}{2}\right)=\frac{(-)^{f} V_{H^{1}}}{2 n}\left(\frac{1}{12}\left(2-6 f+3 f^{2}\right)+\frac{(f-1) n \mu_{\mathrm{E}}}{2 \pi}+\frac{n^{2} \mu_{\mathrm{E}}^{2}}{4 \pi^{2}}\right)$.

For fermions, the linear term in $\mu_{\mathrm{E}}$ vanishes, as expected. However, for bosons there is a linear $\mu_{\mathrm{E}}$ term despite the fact that the sum is explicitly even. This term appears from a term $n \mu_{\mathrm{E}} \ln \left(n \mu_{\mathrm{E}}\right)-n \mu_{\mathrm{E}} \ln \left(-n \mu_{\mathrm{E}}\right)$ in the expansion of the poly-log in $\mu_{\mathrm{E}}$. This suggests that we are actually taking the absolute value of the linear term. This can be readily confirmed by computing the sum numerically. As a result, the free energy has a diverging slope at $\mu_{\mathrm{E}}=0$, suggesting a phase transition there. There are also phase transitions whenever $\mu_{\mathrm{E}} n$ is an integer, as noted above. At precisely $\mu_{\mathrm{E}} n=1 / 2$, the first derivative w.r.t. $\mu_{\mathrm{E}}$ jumps from zero to $V_{H^{1}}$. 
The $\mu_{\mathrm{E}}^{2}$ term cancels out in the Rényi entropy (since it is linear in $n$ ) for $\mu_{\mathrm{E}} n<1 / 2$. The result for a Dirac fermion is

$$
S_{n}^{f}=\frac{c}{6}\left(1+\frac{1}{n}\right) V_{H^{1}}
$$

Note that $c=1 / 2$ for a single Majorana fermion, but this should be doubled for a charged fermion. This reproduces the result obtained via the twist operator method in the main text.

For bosons we obtain instead

$$
S_{n}^{b}=c\left(\frac{1}{6}\left(1+\frac{1}{n}\right)-\frac{\left|\mu_{\mathrm{E}}\right|}{2 \pi}\right) V_{H^{1}},
$$

Again, $c=1$ for a real boson, which must be doubled for a charged boson. One might worry that the result for bosons does not appear to agree with that of fermions given that they are related by bosonization in $1+1$ dimensions. We note however that via bosonization of $U(1)$ charged fermions, the corresponding bosons transform by translation, and thus should instead satisfy the following boundary condition : $\phi(\tau+$ $2 \pi)=\phi(\tau)+n \mu_{\mathrm{E}}$. Therefore, our computation for charged bosons is not related to charged fermions by bosonization. Another point to note is that with the absolute sign, the bosonic result is not analytic even for arbitrarily small $\mu_{\mathrm{E}}$, such that it does not analytically continue to the complex plane, as in the case for fermions.

\section{A.1.2 $d=4$}

Let us work out one more example where there is non-trivial $\mu_{\mathrm{E}}$ dependence. At $d=3+1$, the main difference is the heat kernel for both bosons and fermions on $H^{3}$. For bosons, the equal-point heat kernel is

$$
e^{-m_{s} t} K_{H^{3}}^{b}(0, t)=\frac{1}{(4 \pi t)^{3 / 2}},
$$

where we have substituted in the conformal mass of the scalar. For a Dirac fermion, the heat kernel is

$$
K_{H^{3}}^{f}(0, t)=4 \frac{1}{(4 \pi t)^{3 / 2}}\left(1+\frac{t}{2}\right)
$$

Following the same steps as in $d=3+1$, and again ignoring the $m=0$ term, the Rényi entropy becomes, for bosons :

$$
\begin{aligned}
S_{n} & =\operatorname{tr}(1) V_{H^{3}} \sum_{m \in \mathbb{Z}_{+}} \frac{n^{4} \cos \left(m \mu_{\mathrm{E}}\right)-\cos \left(m n \mu_{\mathrm{E}}\right)}{8 m^{4} \pi^{5}(n-1) n^{3}} \\
& =-\frac{1}{8 \pi^{5}(n-1) n^{3}}\left(-n^{4}\left(\operatorname{Li}_{4}\left(e^{-i \mu_{\mathrm{E}}}\right)+\operatorname{Li}_{4}\left(e^{i \mu_{\mathrm{E}}}\right)\right)+\right. \\
& \left.\operatorname{Li}_{4}\left(e^{-i n \mu_{\mathrm{E}}}\right)+\operatorname{Li}_{4}\left(e^{i n \mu_{\mathrm{E}}}\right)\right)
\end{aligned}
$$


where $\operatorname{tr}(1)=2$ for a pair of real bosons (which together form a complex $U(1)$ charged boson ). The above combinations of poly-logs again admit a representation in terms of the Bernoulli polynomial. Altogether we have

$$
S_{n}^{b}=\frac{V_{H^{3}}}{2 \pi}\left(\frac{1+n+n^{2}+n^{3}}{180 n^{3}}-\frac{(n+1) \mu_{\mathrm{E}}^{2}}{24 \pi^{2} n}+\frac{\mu_{\mathrm{E}}^{3}}{24 \pi^{3}}\right)
$$

Again we are left with a $\mu_{\mathrm{E}}^{3}$ term that is odd in $\mu_{\mathrm{E}}$, and we argue that this term should be enclosed inside an absolute sign since our summation is even. As a result, once again we lose analyticity even for arbitrarily small values of $\mu_{\mathrm{E}}$.

Now let us also look at the corresponding result for fermions. The Rényi entropy is

$$
\begin{aligned}
S_{n}^{f} & =V_{H^{3}} \sum_{m \in \mathbb{Z}_{+}} \frac{(-1)^{m}\left(2+m^{2} \pi^{2} n^{2}\right) \cos \left(m n \mu_{\mathrm{E}}\right)}{m^{4} 4 \pi^{5} n^{3}} \\
& =V_{H^{3}} \frac{(1+n)\left(7+n^{2}\left(37-120 \mu_{\mathrm{E}}^{2}\right)\right.}{1440 \pi n^{3}}
\end{aligned}
$$

which interestingly, is again automatically even in $\mu_{\mathrm{E}}$, and that for purely imaginary $\mu_{\mathrm{E}}$, is positive definite. We note that the above expression is not positive definite in $\mu_{\mathrm{E}}$. We find that for sufficiently large value of $\mu_{\mathrm{E}}$ while within the interval $n \mu_{\mathrm{E}}<1 / 2$ that the above expression can turn negative. This is as expected since the trace

$$
\operatorname{tr} \rho^{n}=\operatorname{tr}\left(e^{-n\left(H-i \mu_{\mathrm{E}} Q\right)}\right)
$$

is not necessarily positive definite quantity. When $\mu_{\mathrm{E}}$ is purely imaginary, we return to the usual thermodynamic chemical potential and the trace should be positive definite. Note that the Rényi entropy for the fermions, which admit analytic continuation for small values of $\mu_{\mathrm{E}}$, is indeed positive definite when $\mu_{\mathrm{E}}$ is purely imaginary.

Remark: $d=2+1$. Here the complication is the more complicated form of the heat kernel in $H^{2}$. Because of that, it doesn't have a neat analytic result, but one can evaluate these results numerically. We find precise agreement with the calculation obtained on a sphere in later sections, and we will not repeat the details here.

\section{A.2 Wavefunctions on $S^{1} \times H^{d-1}$}

We can reproduce the heat kernel results by analyzing the wave functions on the hyperbolic space. This method was used in [10] to study the Rényi entropy of the free theories without the chemical potential. In this subsection, we generalize [10] to include the chemical potential. 


\section{A.2.1 Free scalar field}

We consider a free boson on a $S^{1} \times H^{d-1}$ with $H^{d-1}$ radius $R$

$$
S=\int d^{d} r \sqrt{g}\left(\left|\partial_{\mu} \phi\right|^{2}+M^{2}|\phi|^{2}\right)
$$

where $M$ is the conformal mass. The metric is

$$
d s^{2}=d \theta^{2}+R^{2}\left(d \eta^{2}+\sinh ^{2} \eta d \Omega_{d-2}^{2}\right) .
$$

The periodicity of the $S^{1}$ time circle $(\theta)$ is $2 \pi n$. The Wilson loop changes boundary condition around the time circle from $\phi(2 \pi n)=\phi(0)$ to

$$
\phi(2 \pi n)=e^{i n \mu_{\mathrm{E}}} \phi(0) .
$$

The mode function satisfying this boundary condition is

$$
e^{i\left(\frac{m}{n}+\frac{\mu_{\mathrm{E}}}{2 \pi}\right) \theta},
$$

where $m$ is an integer. The eigenvalue of the Laplace operator $-\Delta-M^{2}$ is

$$
\lambda+\left(\frac{m}{n}+\frac{\mu_{\mathrm{E}}}{2 \pi}\right)^{2}, \quad \lambda \geq 0 .
$$

We define the free energy

$$
\begin{aligned}
F_{n} & =\operatorname{Tr} \log \left(-\Delta-M^{2}\right) \\
& =\sum_{m \in \mathbb{Z}} \int_{0}^{\infty} d \lambda \mathcal{D}(\lambda) \log \left(\lambda+\left(\frac{m}{n}+\frac{\mu_{\mathrm{E}}}{2 \pi}\right)^{2}\right) \\
& =\int_{0}^{\infty} d \lambda \mathcal{D}(\lambda)\left(2 \pi n \sqrt{\lambda}+\log \left(1-2 \cos \left(n \mu_{\mathrm{E}}\right) e^{-2 \pi n \sqrt{\lambda}}+e^{-4 \pi n \sqrt{\lambda}}\right)\right)
\end{aligned}
$$

where $\mathcal{D}(\lambda)$ is the density of states. In the last equation, we used the following formula for the regularized sum

$$
\sum_{k \in \mathbb{Z}} \log \left(\frac{(k+\alpha)^{2}}{n^{2}}+a^{2}\right)=\log [2 \cosh (2 \pi n|a|)-2 \cos (2 \pi \alpha)] .
$$

In the case of scalar bosons, the density of states $\mathcal{D}(\lambda)$ on $H^{d-1}$ is given by [35]

$$
\mathcal{D}(\lambda) d \lambda=\frac{\operatorname{vol}\left(H^{d-1}\right)}{(4 \pi)^{\frac{d-1}{2}} \Gamma\left(\frac{d-1}{2}\right)} \frac{\left|\Gamma\left(i \sqrt{\lambda}+\frac{d-2}{2}\right)\right|^{2}}{\sqrt{\lambda}|\Gamma(i \sqrt{\lambda})|^{2}} d \lambda .
$$


The explicit forms for low dimensions are

$$
\begin{aligned}
& d-1=1 ; \quad \mathcal{D}(\lambda) d \lambda=\frac{\operatorname{vol}\left(H^{1}\right)}{2 \pi \sqrt{\lambda}} d \lambda \\
& d-1=2 ; \quad \mathcal{D}(\lambda) d \lambda=\frac{\operatorname{vol}\left(H^{2}\right)}{4 \pi} \operatorname{th}(\pi \sqrt{\lambda}) d \lambda \\
& d-1=3 ; \quad \mathcal{D}(\lambda) d \lambda=\frac{\operatorname{vol}\left(H^{3}\right)}{(2 \pi)^{2}} \sqrt{\lambda} d \lambda
\end{aligned}
$$

$\operatorname{vol}(H)$ is the regularized volume of the hyperbolic space.

The first term in (A.33) is divergent and needs a regularization. However, it will

not contribute to the Rényi entropy since it linearly depends on $n$. One can show that this integration reproduces the heat kernel results.

\section{A.2.2 Free Dirac fermion}

We also consider a free Fermion

$$
S=\int d^{d} x \sqrt{g} \bar{\psi}(i \not D) \psi
$$

The free energy is

$$
F_{n}=-\operatorname{Tr} \log (i \not D)
$$

In the presence of the Wilson loop, the boundary condition of $\psi$ along the time circle changes from $\psi(2 \pi n)=-\psi(0)$ to

$$
\psi(2 \pi n)=-e^{i n \mu_{\mathrm{E}}} \psi(0)
$$

So the eigenfunction along $\theta$ is $e^{i m \theta / n}$ with

$$
m \in \mathbb{Z}+\frac{1}{2}+\frac{n \mu_{\mathrm{E}}}{2 \pi}
$$

The eigenvalue spectrum of $(i \not D)$ is

$$
\pm \sqrt{\lambda^{2}+\frac{m^{2}}{n^{2}}}
$$

The free energy is

$$
F_{n}=-\frac{1}{2} \sum_{m \in \mathbb{Z}+\frac{1}{2}} \int_{0}^{\infty} d \lambda \mathcal{D}(\lambda) \log \left(\lambda^{2}+\frac{m^{2}}{n^{2}}\right)
$$




$$
=-\frac{1}{2} \int_{0}^{\infty} d \lambda \mathcal{D}(\lambda) \log \left(2 \cosh (2 \pi n \lambda)+2 \cos \left(n \mu_{\mathrm{E}}\right)\right)
$$

As before, we used (A.34) in the last equation.

The density of states $\mathcal{D}(\lambda)$ in $d-1$ dimensions is [35]

$$
\frac{\mathcal{D}(\lambda)}{\operatorname{vol}\left(H^{d-1}\right)}=\left(\frac{\Gamma\left(\frac{d-1}{2}\right) 2^{d-4}}{\pi^{(d-1) / 2+1}} 2^{\left[\frac{(d-1)}{2}\right]}\right) \frac{2^{4-2(d-1)}}{(\Gamma((d-1) / 2))^{2}} \cosh (\pi \lambda)\left|\Gamma\left(i \lambda+\frac{(d-1)}{2}\right)\right|^{2} .
$$

Here, $\mathcal{D}(\lambda)$ is normalized so that the spinor $\zeta$-function per unit volume is given by

$$
\operatorname{tr}\left(-\not D^{2}+m^{2}\right)^{-s}=\int_{0}^{\infty}\left(\lambda^{2}+m^{2}\right)^{-s} \frac{\mathcal{D}(\lambda)}{\operatorname{vol}\left(H^{d-1}\right)} d \lambda
$$

For odd $d-1$, it is

$$
\frac{\mathcal{D}(\lambda)}{\operatorname{vol}\left(H^{d-1}\right)}=\frac{\pi}{2^{2(d-3)}(\Gamma((d-1) / 2))^{2}} \prod_{j=\frac{1}{2}}^{(d-3) / 2}\left(\lambda^{2}+j^{2}\right)
$$

and for even $d-1$

$$
\frac{\mathcal{D}(\lambda)}{\operatorname{vol}\left(H^{d-1}\right)}=\frac{\pi \lambda \operatorname{coth}(\pi \lambda)}{2^{2(d-3)}(\Gamma((d-1) / 2))^{2}} \prod_{j=1}^{(d-3) / 2}\left(\lambda^{2}+j^{2}\right)
$$

The explicit forms for low dimensions are

$$
\begin{array}{ll}
d-1=1 ; & \mathcal{D}(\lambda) d \lambda=\frac{\operatorname{vol}\left(H^{1}\right)}{\pi} d \lambda \\
d-1=2 ; & \mathcal{D}(\lambda) d \lambda=\frac{\operatorname{vol}\left(H^{2}\right)}{\pi} \lambda \operatorname{coth}(\pi \lambda) d \lambda \\
d-1=3 ; & \mathcal{D}(\lambda) d \lambda=\operatorname{vol}\left(H^{3}\right)\left(\lambda^{2}+\frac{1}{4}\right) d \lambda
\end{array}
$$

The first term in (A.42) diverges and needs a regularization, while the second term is finite. We can regularize the divergence using zeta function regularization or flat space subtraction. In any case, it doesn't contribute to the Rényi entropy since it is linear in $n$. The final result agrees with the twist operator computation (Sec 2.4), the heat kernel computation (Appendix A.1) and the wave function computation on a sphere (Appendix A.3) 


\section{A.3 Wavefunctions on $S^{d}$}

Another convenient way of computing the Rényi entropy of CFT is to map onto a sphere. Let us consider a scalar field on $S^{3}$. The metric is

$$
d s^{2}=\cos ^{2} \theta d \tau^{2}+d \theta^{2}+\sin ^{2} \theta d \phi^{2}
$$

with $0 \leq \tau<2 \pi n, 0 \leq \phi<2 \pi$, and $0 \leq \theta<\pi / 2$. Because of the periodicity of $\tau$, there is a conical singularity at $\cos \theta=0$. We can do the heat kernel analysis on the sphere in a similar way to the hyperbolic case. However, we need to set a regularity condition at the conical singularity. An alternative way to compute the free energy is to look at the wave functions and their eigenvalues directly. The analysis below is a generalization of [10] and we cite several results from their paper.

\section{A.3.1 Free scalar field}

The free energy of the free scalar field on the sphere is

$$
F_{n}=\operatorname{tr} \log \left(-\Delta+\frac{\mathcal{R}}{8}\right)
$$

where $\mathcal{R}=6$ for $3 d$ case. We assume that the eigenfunction of the Laplacian takes the form $f(\theta) e^{i m_{\tau} \tau+i m_{\phi} \phi}$. The function $f(\theta)$ obeys the following equation

$$
f^{\prime \prime}(\theta)+2 \cot \theta f^{\prime}(\theta)-\left(\frac{m_{\tau}^{2}}{\cos ^{2} \theta}+\frac{m_{\phi}^{2}}{\sin ^{2} \theta}\right) f(\theta)=\lambda f(\theta) .
$$

From the regularity of $f(\theta)$, the eigenvalue $\lambda$ is fixed to

$$
\lambda=-s(s+2), \quad s=\left|m_{\tau}\right|+\left|m_{\phi}\right|+2 a, \quad a \in \mathbb{N}
$$

The periodicity of $\phi$ requires $m_{\phi}$ to be quantized in $\mathbb{Z}$. In the presence of the Wilson loop, the boundary condition of $\phi$ becomes

$$
\phi(2 \pi n)=e^{i n \mu_{\mathrm{E}}} \phi(0)
$$

Therefore, $m_{\tau}$ is quantized in $\frac{\mathbb{Z}}{n}+\frac{\mu_{\mathrm{E}}}{2 \pi}$.

Let us denote

$$
m_{\tau}=\frac{\alpha}{n}+\frac{\mu_{\mathrm{E}}}{2 \pi}, \quad m_{\phi}=\beta, \quad(\alpha, \beta \in \mathbb{Z})
$$

and

$$
\alpha=k n+p, \quad k \in \mathbb{Z}, \quad p \in[0, n-1] .
$$


The free energy (A.49) is

$$
F_{n}=\sum_{k=-\infty}^{\infty} \sum_{p=0}^{n-1} \sum_{\beta=-\infty}^{\infty} \sum_{a=0}^{\infty} \log \left(s(s+2)+\frac{3}{4}\right)
$$

with

$$
s=\left|k+\frac{p}{n}+\frac{\mu_{\mathrm{E}}}{2 \pi}\right|+|\beta|+2 a .
$$

We want to count the degeneracied for a given value of $s$. Let us first assume that $\mu_{\mathrm{E}}$ satisfies $0 \leq \frac{\mu_{\mathrm{E}}}{2 \pi}<\frac{1}{q}$. Then the degeneracy for

$$
s=m+\frac{p}{n}+\frac{\mu_{\mathrm{E}}}{2 \pi}, \quad m-\frac{p}{n}-\frac{\mu_{\mathrm{E}}}{2 \pi}+1 \quad(p \in[0, n-1], \quad m \in \mathbb{N}),
$$

is

$$
\frac{(m+1)(m+2)}{2} .
$$

Therefore, the free energy is

$$
\begin{aligned}
F_{n} & =\sum_{p=0}^{q} \sum_{m=0}^{\infty}\left[\frac{(m+1)(m+2)}{2}\left\{\log \left(\left(m+\frac{p}{q}+\frac{\mu_{\mathrm{E}}}{2 \pi}+1\right)^{2}-\frac{1}{4}\right)+\log \left(\left(m-\frac{p}{q}-\frac{\mu_{\mathrm{E}}}{2 \pi}+2\right)^{2}-\frac{1}{4}\right)\right\}\right] \\
& =\sum_{p=0}^{q} \sum_{a=a_{1}}^{a_{4}}\left[-\frac{1}{2}\left(\zeta^{\prime}(-2, a)+(3-2 a) \zeta^{\prime}(-1, a)+\left(a^{2}-3 a+2\right) \zeta^{\prime}(0, a)\right)\right]
\end{aligned}
$$

where $\zeta^{\prime}(s, a)=\frac{\partial \zeta(s, a)}{\partial s}$ and

$a_{1}=\left(\frac{p}{n}+\frac{\mu_{\mathrm{E}}}{2 \pi}+\frac{1}{2}\right), \quad a_{2}=\left(\frac{p}{n}+\frac{\mu_{\mathrm{E}}}{2 \pi}+\frac{3}{2}\right), \quad a_{3}=\left(-\frac{p}{n}-\frac{\mu_{\mathrm{E}}}{2 \pi}+\frac{3}{2}\right), \quad a_{4}=\left(-\frac{p}{n}-\frac{\mu_{\mathrm{E}}}{2 \pi}+\frac{5}{2}\right)$.

The expansions near $\mu_{\mathrm{E}}=0$ are

$$
\begin{aligned}
F_{1}= & \frac{\log 2}{4}-\frac{3 \zeta(3)}{8 \pi^{2}} \\
& -\frac{\mu_{\mathrm{E}}^{2}}{96 \pi^{2}}\left(2 \left(-12 \zeta^{(1,1)}\left(-1, \frac{1}{2}\right)-24 \zeta^{(1,1)}\left(-1, \frac{3}{2}\right)-12 \zeta^{(1,1)}\left(-1, \frac{5}{2}\right)+3 \zeta^{(1,2)}\left(-2, \frac{1}{2}\right)\right.\right. \\
& +6 \zeta^{(1,2)}\left(-2, \frac{3}{2}\right)+3 \zeta^{(1,2)}\left(-2, \frac{5}{2}\right)+6 \zeta^{(1,2)}\left(-1, \frac{1}{2}\right)-6 \zeta^{(1,2)}\left(-1, \frac{5}{2}\right) \\
& \left.+28-36 \log 2+6 \log 3)+3 \pi^{2}\right)+\mathcal{O}\left(\mu_{\mathrm{E}}^{4}\right), \\
F_{2}= & \frac{\log 2}{4}+\frac{\zeta(3)}{8 \pi^{2}}
\end{aligned}
$$




$$
\begin{aligned}
& +\frac{\mu_{\mathrm{E}}^{2}}{4 \pi^{2}}\left(\frac { 1 } { 1 2 } \left(12 \zeta^{(1,1)}\left(-1, \frac{1}{2}\right)+24 \zeta^{(1,1)}(-1,1)+24 \zeta^{(1,1)}\left(-1, \frac{3}{2}\right)+24 \zeta^{(1,1)}(-1,2)+\right.\right. \\
& 12 \zeta^{(1,1)}\left(-1, \frac{5}{2}\right)-3 \zeta^{(1,2)}\left(-2, \frac{1}{2}\right)-6 \zeta^{(1,2)}(-2,1)-6 \zeta^{(1,2)}\left(-2, \frac{3}{2}\right) \\
& -6 \zeta^{(1,2)}(-2,2)-3 \zeta^{(1,2)}\left(-2, \frac{5}{2}\right)-6 \zeta^{(1,2)}\left(-1, \frac{1}{2}\right)-6 \zeta^{(1,2)}(-1,1)+6 \zeta^{(1,2)}(-1,2) \\
& \left.\left.+6 \zeta^{(1,2)}\left(-1, \frac{5}{2}\right)-40-6 \log (3)+12 \log (16 \pi)\right)-\frac{\pi^{2}}{8}\right)+\mathcal{O}\left(\mu_{\mathrm{E}}^{4}\right) .
\end{aligned}
$$

Each of the functions $\zeta^{(1,1)}, \zeta^{(1,2)}$ etc has some subtlety in evaluation. We may always go back to the expression (A.59) when we evaluate the free energy explicitly. The leading terms agrees with the results in [10].

The expression (A.59) is not valid for $\frac{\mu_{\mathrm{E}}}{2 \pi}>\frac{1}{n}$. In this case, there is a number $p_{1} \leq n-1$ satisfying

$$
-1+\frac{p_{1}}{n}+\frac{\mu_{\mathrm{E}}}{2 \pi}<0, \quad-1+\frac{p_{1}+1}{n}+\frac{\mu_{\mathrm{E}}}{2 \pi}<0 .
$$

By using this number, the eigenvalues and their degeneracies become

$$
\begin{aligned}
\frac{(m+1)(m+2)}{2} & \text { for } \quad s=m+\frac{p}{n}+\frac{\mu_{\mathrm{E}}}{2 \pi}, \quad m-\frac{p}{n}-\frac{\mu_{\mathrm{E}}}{2 \pi}+2(p \in[0, n-1]), \\
(m+1) & \text { for } \quad s=m-\frac{p}{n}-\frac{\mu_{\mathrm{E}}}{2 \pi}+1 \quad\left(p \in\left[0, p_{1}\right]\right), \\
(m+1) & \text { for } \quad s=m+\frac{p}{n}+\frac{\mu_{\mathrm{E}}}{2 \pi}-1 \quad\left(p \in\left[p_{1}+1, n-1\right]\right) .
\end{aligned}
$$

The free energy is

$$
\begin{aligned}
F_{n}= & \sum_{p=0}^{n-1} \sum_{m=0}^{\infty} \frac{(m+1)(m+2)}{2}\left(\log \left(\left(m+\frac{p}{n}+\frac{\mu_{\mathrm{E}}}{2 \pi}+1\right)^{2}-\frac{1}{4}\right)+\log \left(\left(m-\frac{p}{n}-\frac{\mu_{\mathrm{E}}}{2 \pi}+3\right)^{2}-\frac{1}{4}\right)\right) \\
& +\sum_{p=0}^{p_{1}} \sum_{m=0}^{\infty}(m+1)\left(\log \left(\left(m-\frac{p}{n}-\frac{\mu_{\mathrm{E}}}{2 \pi}+2\right)^{2}-\frac{1}{4}\right)\right) \\
& +\sum_{p=p_{1}+1}^{n-1} \sum_{m=0}^{\infty}(m+1)\left(\log \left(\left(m+\frac{p}{n}+\frac{\mu_{\mathrm{E}}}{2 \pi}\right)^{2}-\frac{1}{4}\right)\right) \\
= & \sum_{p=0}^{n} \sum_{a=b_{1}}^{b_{4}}\left(-\frac{1}{2}\left(\zeta^{\prime}(-2, a)+(3-2 a) \zeta^{\prime}(-1, a)+\left(a^{2}-3 a+2\right) \zeta^{\prime}(0, a)\right)\right) \\
& +\sum_{p=0}^{p_{1}} \sum_{a=c_{1}}^{c_{2}}(-(\zeta(-1, a)+(1-a) \zeta(0, a)))+\sum_{p=p_{1}+1}^{n-1} \sum_{a=c_{3}}^{c_{4}}(-(\zeta(-1, a)+(1-a) \zeta(0, a)))(\mathrm{A} .65)
\end{aligned}
$$

where

$$
b_{1}=\left(\frac{p}{q}+\frac{\mu_{\mathrm{E}}}{2 \pi}+\frac{1}{2}\right), \quad b_{2}=\left(\frac{p}{q}+\frac{\mu_{\mathrm{E}}}{2 \pi}+\frac{3}{2}\right), \quad b_{3}=\left(-\frac{p}{q}-\frac{\mu_{\mathrm{E}}}{2 \pi}+\frac{5}{2}\right), \quad b_{4}=\left(-\frac{p}{q}-\frac{\mu_{\mathrm{E}}}{2 \pi}+\frac{7}{2}\right),
$$


and

$$
c_{1}=\left(-\frac{p}{q}-\frac{\mu_{\mathrm{E}}}{2 \pi}+\frac{3}{2}\right), \quad c_{2}=\left(-\frac{p}{q}-\frac{\mu_{\mathrm{E}}}{2 \pi}+\frac{5}{2}\right), \quad c_{3}=\left(\frac{p}{q}+\frac{\mu_{\mathrm{E}}}{2 \pi}-\frac{1}{2}\right), \quad c_{4}=\left(\frac{p}{q}+\frac{\mu_{\mathrm{E}}}{2 \pi}+\frac{1}{2}\right) .
$$

We show the numerical result in Fig.6. It is remarkable that the function is smooth around $\mu_{\mathrm{E}}=\frac{1}{n}$.

$F_{\mathrm{O}}\left(\mu_{E}\right) / F_{\mathrm{O}}(\mathrm{M}$

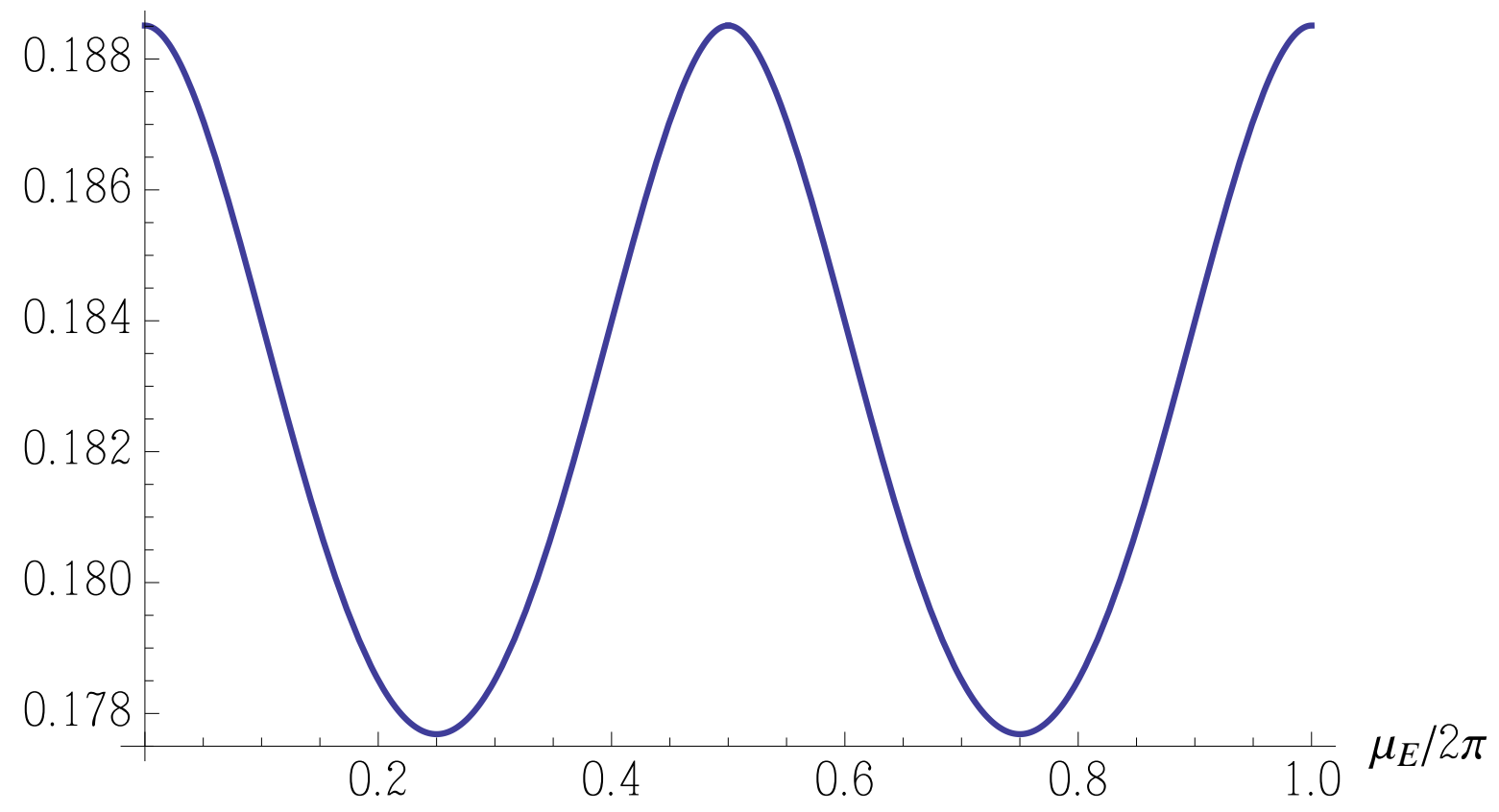

Figure 6: Boson free energy $n=2$

\section{A.3.2 Free Dirac fermion}

Next, we consider a spinor $\psi$ on $S^{3}$. It satisfies the Dirac equation

$$
i \sigma_{a}^{\mu} \gamma^{a}\left(\partial_{\mu} \psi+\frac{1}{4} \omega_{\mu}^{a b} \gamma_{a b} \psi\right)=\lambda \psi
$$

The periodic part of the spinor $\psi$ in $\tau$ and $\phi$ directions can be written as $e^{i m_{\tau} \tau+i m_{\phi} \phi}$. As shown in [10], the regularity condition at $\theta=0$ restricts the allowed eigenvalue $\lambda$. There are four types of eigenvalues:

Two positive $\lambda$

$$
\text { Case } 1 \quad \lambda=m_{\tau}+m_{\phi}+\frac{3}{2}+2 a, \quad m_{\tau} \geq 0, \quad m_{\phi} \geq-\frac{1}{2}-a, \quad a \in \mathbb{N},
$$




$$
\text { Case } 2 \lambda=-m_{\tau}+m_{\phi}+\frac{1}{2}+2 a, \quad m_{\tau}<0, \quad m_{\phi} \geq \frac{1}{2}-a, \quad a \in \mathbb{N},
$$

and two negative $\lambda$

$$
\begin{aligned}
& \text { Case } 3 \lambda=-\left(m_{\tau}+m_{\phi}+\frac{1}{2}+2 a\right), \quad m_{\tau} \geq 0, \quad m_{\phi} \geq \frac{1}{2}-a, \quad a \in \mathbb{N}, \\
& \text { Case } 4 \quad \lambda=-\left(-m_{\tau}+m_{\phi}+\frac{3}{2}+2 a\right), \quad m_{\tau}<0, \quad m_{\phi} \geq-\frac{1}{2}-a, \quad a \in \mathbb{N},(\text { A.70 })
\end{aligned}
$$

where

$$
m_{\tau} \in \frac{1}{q}\left(\mathbb{Z}+\frac{1}{2}+\frac{q \mu_{\mathrm{E}}}{2 \pi}\right), \quad m_{\phi} \in \mathbb{Z}+\frac{1}{2}
$$

As before, we first consider the case $0 \leq \frac{n \mu_{\mathrm{E}}}{2 \pi}<\frac{1}{2}$. In this case, the eigenvalues and the degeneracies are

$$
\frac{(k+1)(k+2)}{2} \text { for } \quad \lambda= \pm\left(k+\frac{p}{q}+\frac{1}{2 q}+\frac{\mu_{\mathrm{E}}}{2 \pi}+1\right), \pm\left(k+\frac{p}{q}+\frac{1}{2 q}-\frac{\mu_{\mathrm{E}}}{2 \pi}+1\right)
$$

where $k \in \mathbb{N}$.

The free energy is

$$
\begin{aligned}
F_{n}= & -2 \sum_{p=0}^{q-1} \sum_{k=0}^{\infty} \frac{(k+1)(k+2)}{2} \log \left(k+1+\frac{p}{n}+\frac{1}{2 n}+\frac{\mu_{\mathrm{E}}}{2 \pi}\right) \\
& -2 \sum_{p=0}^{n-1} \sum_{k=0}^{\infty} \frac{(k+1)(k+2)}{2} \log \left(k+1+\frac{p}{n}+\frac{1}{2 n}-\frac{\mu_{\mathrm{E}}}{2 \pi}\right) \\
= & \sum_{p=0}^{q-1} \sum_{a=a_{1}}^{a_{2}}\left(\zeta^{\prime}(-2, a)+(1-2 a) \zeta^{\prime}(-1, a)+a(a-1) \zeta^{\prime}(0, a)\right)
\end{aligned}
$$

where

$$
a_{1}=1+\frac{p}{n}+\frac{1}{2 n}+\frac{\mu_{\mathrm{E}}}{2 \pi}, \quad a_{1}=1+\frac{p}{n}+\frac{1}{2 n}-\frac{\mu_{\mathrm{E}}}{2 \pi}
$$

We show the explicit form of the free energy near $\mu_{\mathrm{E}}=0$.

$$
\begin{aligned}
F_{1} & =\frac{\log 2}{4}+\frac{3 \zeta(3)}{8 \pi^{2}} \\
& +\left(-\log 8-4 \zeta^{(1,1)}\left(-1, \frac{3}{2}\right)+\zeta^{(1,2)}\left(-2, \frac{3}{2}\right)-\frac{1}{4} \zeta^{(1,2)}\left(0, \frac{3}{2}\right)\right) \frac{\mu_{\mathrm{E}}^{2}}{4 \pi^{2}}+\mathcal{O}\left(\mu_{\mathrm{E}}^{4}\right) \\
F_{2} & =\frac{3 \zeta(3)}{32 \pi^{2}}+\frac{3 \log 2}{16}+\frac{G}{2 \pi} \\
& +\left(-9 \log 2+2 \log 3-4 \zeta^{(1,1)}\left(-1, \frac{5}{4}\right)-4 \zeta^{(1,1)}\left(-1, \frac{7}{4}\right)-\zeta^{(1,1)}\left(0, \frac{5}{4}\right)+\zeta^{(1,1)}\left(0, \frac{7}{4}\right)\right.
\end{aligned}
$$




$$
\begin{aligned}
& +\zeta^{(1,2)}\left(-2, \frac{5}{4}\right)+\zeta^{(1,2)}\left(-2, \frac{7}{4}\right)+\frac{1}{2} \zeta^{(1,2)}\left(-1, \frac{5}{4}\right)-\frac{1}{2} \zeta^{(1,2)}\left(-1, \frac{7}{4}\right) \\
& \left.-\frac{3}{16} \zeta^{(1,2)}\left(0, \frac{5}{4}\right)-\frac{3}{16} \zeta^{(1,2)}\left(0, \frac{7}{4}\right)\right) \frac{\mu_{\mathrm{E}}^{2}}{4 \pi^{2}}+\mathcal{O}\left(\mu_{\mathrm{E}}^{4}\right)
\end{aligned}
$$

where $G$ is the Catalan constant. Again, the functions $\zeta^{(1,1)}, \zeta^{(1,2)}$ etc are a formal expression and one may use (A.73) to evaluate the free energy. The leading terms agree with [10]. Notice that only even powers of $\mu_{\mathrm{E}}$ appears in the expansion. The expression (A.73) is not valid for $\mu_{\mathrm{E}}>\frac{1}{2 n}$. In this region there is a number $p_{1}$ which satisfies

$$
-1+\frac{p_{1}}{n}+\frac{1}{2 n}+\frac{\mu_{\mathrm{E}}}{2 \pi}<0, \quad-1+\frac{p_{1}+1}{n}+\frac{1}{2 n}+\frac{\mu_{\mathrm{E}}}{2 \pi} \geq 0 .
$$

Then the eigenvalues, their degeneracies, and the range of $p$ are as follows:

$$
\begin{array}{rlll}
\frac{(k+1)(k+2)}{2} & \text { for } \lambda= \pm\left(k+1+\frac{p}{n}+\frac{1}{2 n}+\frac{\mu_{\mathrm{E}}}{2 \pi}\right), & p \in[0, n-1] \\
\frac{(k+1)(k+2)}{2} & \text { for } \lambda= \pm\left(k+3-\frac{p}{n}-\frac{1}{2 n}-\frac{\mu_{\mathrm{E}}}{2 \pi}\right), & p \in[0, n-1] \\
(k+1) & \text { for } \lambda= \pm\left(k+2-\frac{p}{n}-\frac{1}{2 n}-\frac{\mu_{\mathrm{E}}}{2 \pi}\right), & p \in\left[0, p_{1}\right] \\
(k+1) & \text { for } \lambda= \pm\left(k+\frac{p}{n}+\frac{1}{2 n}+\frac{\mu_{\mathrm{E}}}{2 \pi}\right), \quad p \in\left[p_{1}+1, n-1\right]
\end{array}
$$

where $k \in \mathbb{N}$.

The free energy is

$$
\begin{aligned}
& F_{n}=\sum_{p=0}^{n-1} \sum_{a=a_{1}}^{a_{2}}\left(\zeta^{\prime}(-2, a)+(3-2 a) \zeta^{\prime}(-1, a)+\left(a^{2}-3 a+2\right) \zeta^{\prime}(0, a)\right) \\
& +\sum_{p=0}^{p_{1}} 2\left(\zeta\left(-1, c_{1}\right)+\left(1-c_{1}\right) \zeta\left(0, c_{1}\right)\right)+\sum_{p=p_{1}+1}^{n-1} 2\left(\zeta\left(-1, c_{2}\right)+\left(1-c_{2}\right) \zeta\left(0, c_{2}\right)\right)(A
\end{aligned}
$$

with

$a_{1}=1+\frac{p}{q}+\frac{1}{2 q}+\frac{\mu_{\mathrm{E}}}{2 \pi}, \quad a_{2}=3-\frac{p}{q}-\frac{1}{2 q}-\frac{\mu_{\mathrm{E}}}{2 \pi}, \quad c_{1}=2-\frac{p}{q}-\frac{1}{2 q}-\frac{\mu_{\mathrm{E}}}{2 \pi}, \quad c_{2}=\frac{p}{q}+\frac{1}{2 q}+\frac{\mu_{\mathrm{E}}}{2 \pi}$.

The numerical result is shown in Fig.7. There are phase transitions at $\frac{\mu_{\mathrm{E}}}{2 \pi}=\frac{1}{2 n}+\frac{\mathbb{Z}}{n}$ for the free energy and $\frac{\mu_{\mathrm{E}}}{2 \pi}=\frac{1}{2 n}+\frac{\mathbb{Z}}{n}$ and $\frac{1}{2}+\mathbb{Z}$ for the Rényi entropy.

\section{B Holographic computations for $d=2$}

We now compute the holographic charged Rényi entropies for $d=2$ using a threedimensional bulk dual. In this case there are two interesting bulk duals: EinsteinMaxwell theory and Einstein-Chern-Simons theory. 


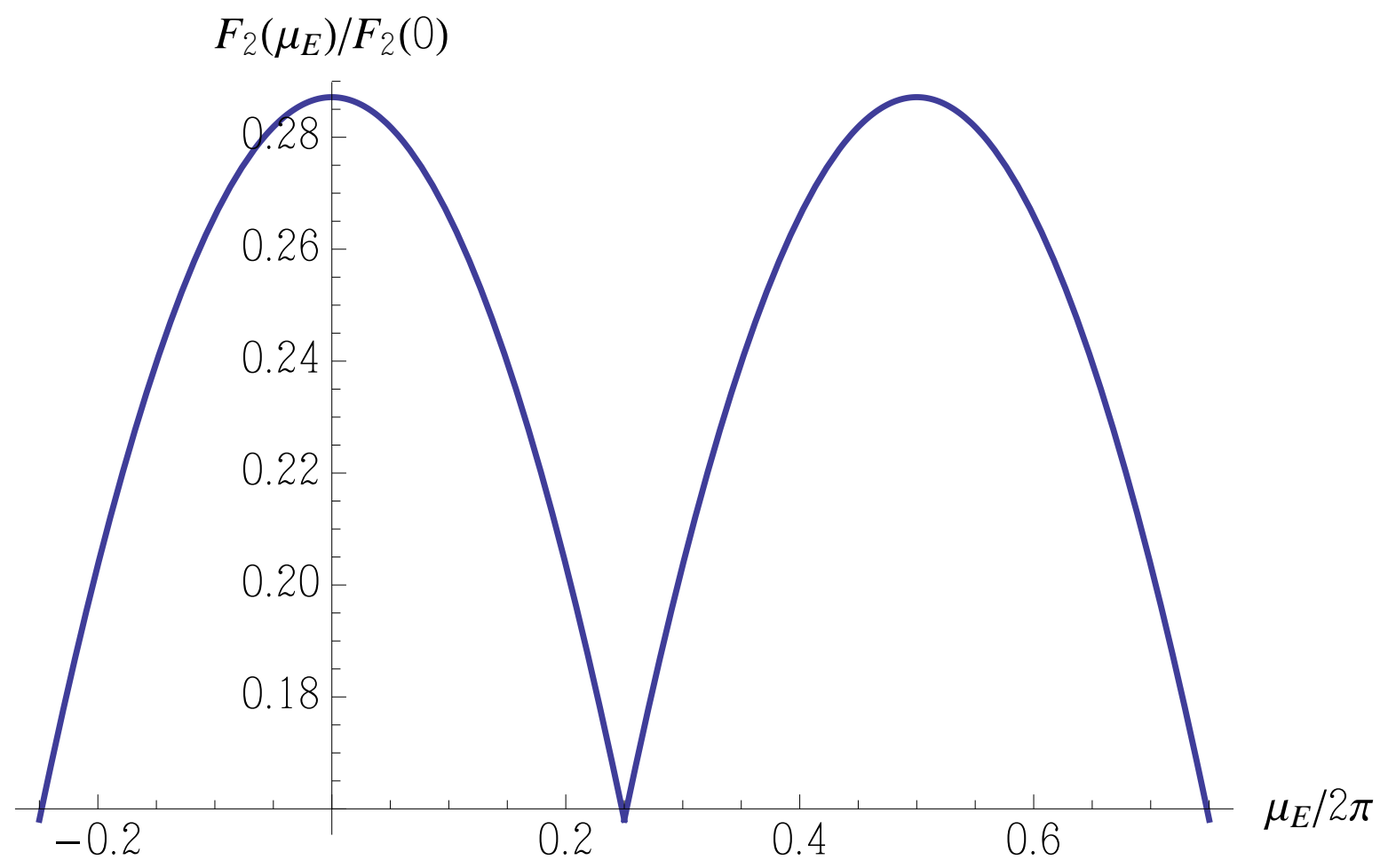

Figure 7: Fermion free energy $n=2$

\section{B.1 Einstein-Maxwell theory}

Starting with the Einstein-Maxwell action in a three-dimensional bulk

$$
I_{E-M}=\frac{1}{2 \ell_{\mathrm{P}}} \int d^{3} x \sqrt{-g}\left(\frac{2}{L^{2}}+\mathcal{R}-\frac{\ell_{*}^{2}}{4} F_{\mu \nu} F^{\mu \nu}\right) .
$$

The charged black hole solution analogous to eq. (3.2) for this theory is [36]

$$
d s^{2}=-f(r) \frac{L^{2}}{R^{2}} d t^{2}+\frac{d r^{2}}{f(r)}+r^{2} d \theta^{2},
$$

with

$$
f(r)=\frac{r^{2}}{L^{2}}-m-\frac{q^{2}}{2} \log \left(\frac{r}{L \sqrt{m}}\right) .
$$

With the above parametrization, the horizon radius is simply $r_{H}=L \sqrt{m}$. Note that the geometry is not asymptotically AdS because of the logarithmic term appearing in $f(r)$. Similar situations were considered in [37-39] and this logarithmic behaviour is 
the signature of broken conformal invariance, even in the UV. The solution for bulk gauge field is

$$
A=-\frac{q L}{\ell_{*} R} \log \left(\frac{r}{L \sqrt{m}}\right) d t .
$$

The integration constant in eq. (B.4) was chosen to ensure that $A=0$ at the horizon. However, because of the logarithmic running of the bulk gauge field, one can not discern the chemical potential and the expectation value of the dual charge density as easily as in section 3 for $d \geq 3$. Following [38, 39], we arbitrarily chose a renormalization scale which will be defined by the radius $r=r_{R}$. Then the chemical potential is given by

$$
\mu=-q \log \frac{r_{H}}{r_{R}}
$$

Hence we find that the chemical potential runs logarithmically with the renormalization scale.

The temperature of the black hole (B.2) is

$$
\begin{aligned}
T=\frac{L f^{\prime}\left(r_{H}\right)}{4 \pi R} & =\frac{r_{H}}{2 \pi R L}\left(1-\frac{L^{2} \mu^{2}}{4 r_{H}^{2} \log \left[r_{H} / r_{R}\right]^{2}}\right) \\
& =T_{0}\left(x-\frac{\mu^{2}}{4 x \log \left(\frac{x L}{r_{R}}\right)^{2}}\right)
\end{aligned}
$$

where as before, we have introduced the parameter $x=r_{H} / L$. The horizon entropy is given by

$$
S=2 \pi r_{H}=2 \pi L x .
$$

The charged Rényi entropy for this solution is again determined with eq. (3.11), however, we note that in this integral, both the chemical potential and the renormalization scale $r_{R}$ are held fixed. The endpoints of the integral are again chosen such that $T\left(x_{1}, \mu, r_{R}\right)=T_{0}$ and $T\left(x_{n}, \mu, r_{R}\right)=T_{0} / n$. Given the form of eq. (B.6), the $x_{n}$ can only be solved numerically for given $\mu$ (and $r_{R}$ ). Combining the previous results, we can write the charged Rényi entropy as follows:

$$
S_{n}(\mu)=\frac{\pi L}{n-1}\left(2 n x_{1}-2 x_{n}+n\left(x_{n}^{2}-x_{1}^{2}\right)+\mu\left(\sqrt{n x_{n}\left(n x_{n}-1\right)}-n \sqrt{x_{1}\left(x_{1}-1\right)}\right)\right) .
$$

In figure 8, we show the behaviour of the charge Rényi entropy as a function of $\mu$ for various values of $n$. For large values of $\mu$, all of the $S_{n}(\mu)$ appear to increase linearly. From figure 9 , we see that in the limit $n \rightarrow \infty, S_{n}(\mu)$ seems to approach a 


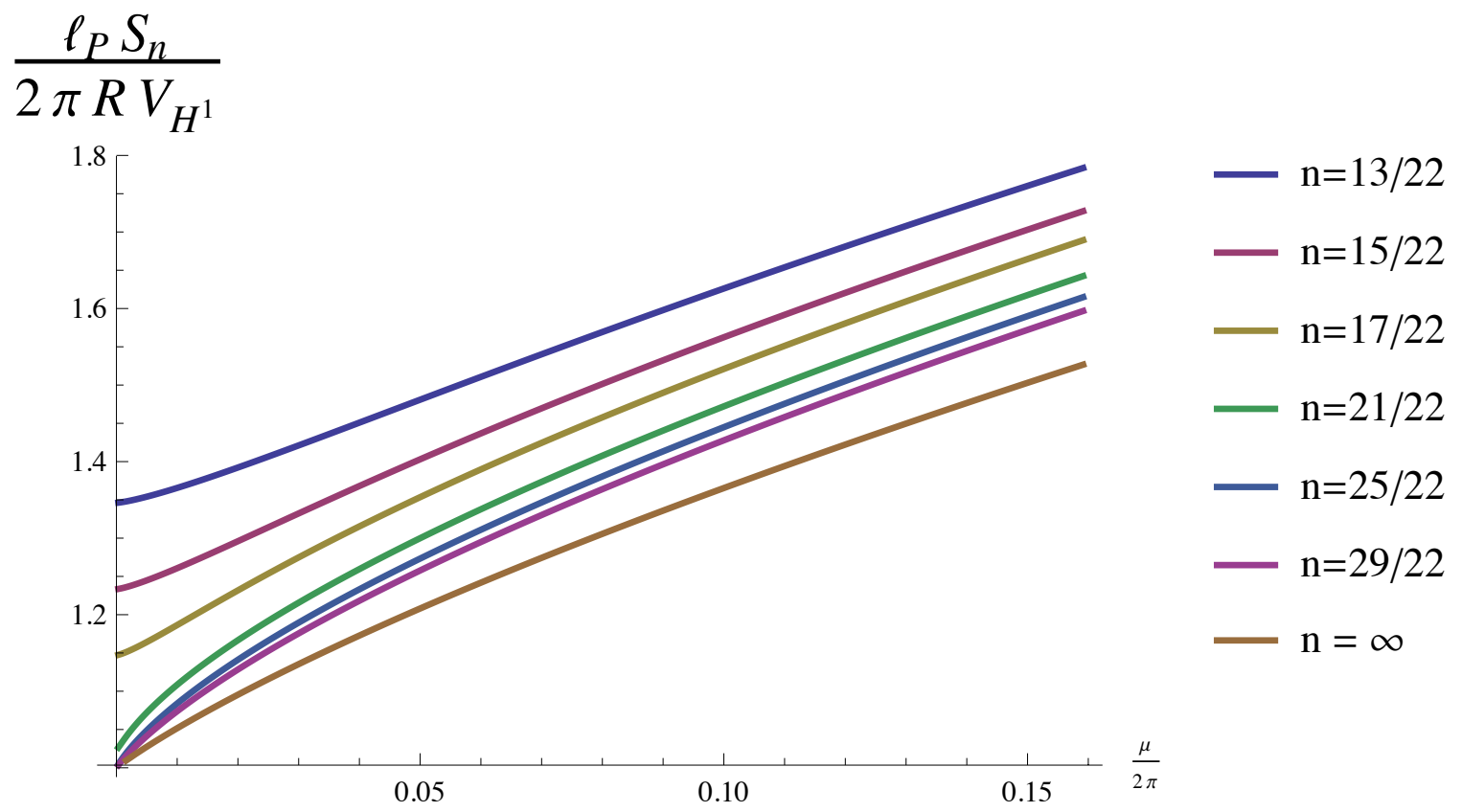

Figure 8: Charged Rényi entropy evaluated for the charged BTZ black hole at various values of $n$, setting $L=r_{R}=1$.

finite asymptotic value (which depends on $\mu$ ), and we have included a plot in figure 8. This may be contrasted with the behaviour in eq. (3.15) for the same limit in higher dimensions. Further, the $n \rightarrow 0$ limit appears to diverge, in agreement with the analogous limit in higher dimensions, as given in eq. (3.15).

It is again interesting to consider imaginary chemical potentials, which is accomplished here by replacing $\mu=i \mu_{\mathrm{E}}$ and $q=i q_{\mathrm{E}}$ in the above results. The horizon radius for which $T=T_{0} / n$ is now given by

$$
x_{n}=\frac{1+\sqrt{1-n^{2} q_{\mathrm{E}}^{2}}}{2 n} .
$$

Clearly, we only have real solutions here when $q_{\mathrm{E}}^{2}<2 / n^{2}$ and so the charge can only take values in a finite range. If we re-write eq. (B.9) as

$$
\left(2 n x_{n}-1\right)^{2}+n^{2} q_{\mathrm{E}}^{2}=1,
$$

we see the horizon radius and the charge can be parameterized by

$$
x_{n}=\frac{1+\cos \phi}{2 n}, \quad q_{\mathrm{E}}=\frac{\sin \phi}{n} .
$$

This is again reminiscent of the free field calculation where analytic continuation is only possible within a finite window for $\mu$. 


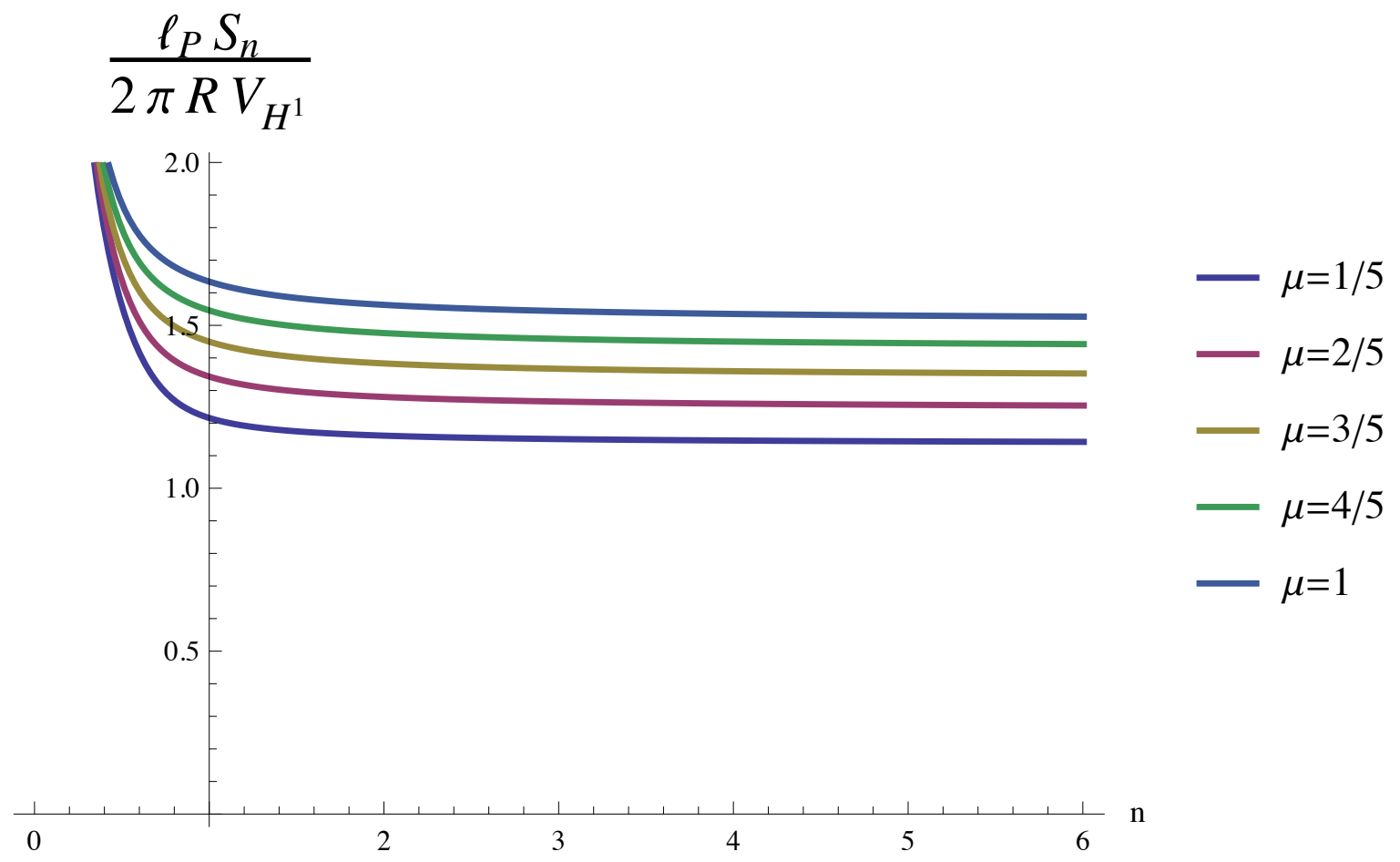

Figure 9: Charged Rényi entropy evaluated for the charged BTZ black hole at various values of $\mu$, setting $L=r_{R}=1$.

\section{B.2 Einstein-Chern-Simons theory}

We first consider the boundary duals of Einstein-Chern-Simons theory. The entropy of the charged BTZ black hole is (see e.g. (6.17) of [40])

$$
S=2 \pi\left(\sqrt{\frac{c}{6}\left(L_{0}-\frac{c}{24}-\frac{1}{4} q^{2}\right)}+\sqrt{\frac{\tilde{c}}{6}\left(\tilde{L}_{0}-\frac{\tilde{c}}{24}-\frac{1}{4} q^{2}\right)}\right) .
$$

where $L_{0}\left(\tilde{L}_{0}\right)$ and $c(\tilde{c})$ are the Virasoro generator and the central charge of the left (right) movers. Here $q$ denotes the charge of the black hole. The expression inside the square root is independent of spectral flow. In terms of the horizon radius $r_{H}$,

$$
L_{0}-\frac{c}{24}-\frac{1}{4} q^{2}=\frac{\pi r_{H}^{2}}{2 \ell_{\mathrm{P}} L}=\tilde{L}_{0}-\frac{\tilde{c}}{24}-\frac{1}{4} q^{2} .
$$

The Hawking temperature of the BTZ black hole is

$$
T=\frac{r_{H}}{2 \pi L^{2}} .
$$


Using $c=12 \pi L / \ell_{\mathrm{P}}$, the entropy is the usual expression

$$
S=2 \pi^{2} \frac{L T c}{3}=\frac{\pi c}{3 n} .
$$

To obtain the Rényi entropy, we integrate $S$. However, since $q$ cancels out, there is no dependence on the chemical potential. We note that this is in complete agreement with the free fermion results at least for sufficiently small $\mu_{E}$. To be more precise, the above statement is as follows: Since the gauge potential does not couple to the metric in Chern-Simons theory, the solution of the equation of motion is a flat connection, $d F=0$, or a constant gauge potential. Without any source term in the bulk, the gauge potential has to be zero. The charge $q$ we mentioned above is a charge along the spatial direction $\theta$. The result suggests that the linear term in the Rényi entropy (2.72) is not protected by a symmetry or an anomaly.

\section{Holographic minutiae}

In this appendix, we present various useful details of the holographic calculations, which are used in section 3 .

\section{C.1 Energy density}

To evaluate the conformal weight of the twist operators using eq. (3.20), we need to evaluated the difference of energy densities: $\mathcal{E}\left(T_{0} / n, \mu\right)-\mathcal{E}\left(T_{0}, \mu=0\right)$. Now in principle, with the introduction of appropriate boundary counterterms [41], one can evaluate each of these energy densities individually. However, since we only need to determine a difference of energy densities, it is simpler to use 'background subtraction,' in which case the counterterms play no role.

To begin, we will denote the metric of a surface of constant $r$ by $\gamma_{\mu \nu}=g_{\mu \nu}-$ $\delta_{\mu r} \delta_{\nu r} / g^{r r}$, and the boundary hyperbolic metric (2.9) by $\hat{\gamma}$. Following [42], we write the boundary stress tensor

$$
\tau_{a b}=\frac{2}{\sqrt{-\gamma}} \frac{\delta I}{\delta \gamma^{a b}}=\frac{1}{\ell_{\mathrm{P}}^{d-1}}\left(\gamma_{a b} K_{c}^{c}-K_{a b}\right),
$$

where $K_{a b}$ is the extrinsic curvature taken on a regulator surface at some constant radius $r$. To leading order as $r \rightarrow \infty$,

$$
\tau_{00}\left(T_{0} / n, \mu\right)-\tau_{00}\left(T_{0}, \mu=0\right)=\frac{(d-1) L m}{2 \ell_{\mathrm{P}}^{d-1} R^{2} r^{d-2}},
$$


where $m$ is given by (3.6). We can then evaluate the energy density of the boundary field theory with [43]

$$
\begin{aligned}
& \sqrt{-\hat{\gamma}} \hat{\gamma}^{00}\left(T_{\tau \tau}\left(T_{0} / n, \mu\right)-T_{\tau \tau}\left(T_{0}, \mu=0\right)\right) \\
& =\lim _{r \rightarrow \infty} \sqrt{-\gamma} \gamma^{00}\left(\tau_{00}\left(T_{0} / n, \mu\right)-\tau_{00}\left(T_{0}, \mu=0\right)\right) .
\end{aligned}
$$

Now using the notation $\mathcal{E}=T_{\tau \tau}$ from the main text, this equation yields the desired difference of energy densities:

$$
\begin{aligned}
\mathcal{E}\left(T_{0} / n, \mu\right) & -\mathcal{E}\left(T_{0}, \mu=0\right)=\frac{(d-1) L m}{2 \ell_{\mathrm{P}}^{d-1} R^{d}} \\
= & \frac{(d-1)}{2 R^{d}} \frac{L^{d-1}}{\ell_{\mathrm{P}}^{d-1}}\left(x_{n}^{d-2}\left(x_{n}^{2}-1\right)+\frac{q^{2}}{\left(L r_{H}\right)^{d-2}}\right) .
\end{aligned}
$$

\section{C.2 Charge density}

In order to determine the magnetic response for our holographic model, we must evaluate the charge density $\rho(n, \mu)=\left\langle J_{\tau}\right\rangle$ in eq. (3.24). Of course, the standard AdS/CFT dictionary indicates this expectation value is given by the normalizable component of the gauge field (3.4), i.e., $\left\langle J_{\tau}\right\rangle \propto q$. However, to make precise comparisons with the expansion coefficients derived in section 2.3, we need the exact normalization of the current. We evaluate the latter here with a simple thermodynamic analysis.

Recall that the first law of thermodynamics of our ensemble is given by

$$
d \mathcal{E}=T d s+\frac{\mu}{2 \pi R} d \rho
$$

where $\mathcal{E}, s$ and $\rho$ denote the energy, entropy and charge densities respectively. Hence if the entropy density is held fixed, it follows that

$$
\frac{\mu}{2 \pi R}=\left(\frac{\partial \mathcal{E}}{\partial \rho}\right)_{s}
$$

Now as observed above, we have $\rho(n, \mu)=\alpha q$ where $\alpha$ is some numerical factor which we aim to determine. From eq. (3.9), we can see that holding the entropy density fixed is equivalent to holding $r_{H}$ constant. Hence it follows that

$$
\begin{aligned}
\alpha & =\frac{2 \pi R}{\mu}\left(\frac{\partial \mathcal{E}}{\partial q}\right)_{r_{H}} \\
& =\sqrt{\frac{(d-1)(d-2)}{2}} \frac{\ell_{*}}{\ell_{\mathrm{P}}^{d-1} R^{d-1}}
\end{aligned}
$$

where up to a constant independent of $q, \mathcal{E}$ is given by eq. (C.4). Therefore, our final result for the charge density is

$$
\rho(n, \mu)=\frac{(d-2) x_{n}^{d-2}}{4 \pi R^{d-1}} \frac{L^{d-3} \ell_{*}^{2}}{\ell_{\mathrm{P}}^{d-1}} \mu .
$$




\section{C.3 Boundary CFT parameters}

Here, we provide the values of various parameters, i.e., $C_{V}, \hat{c}, \hat{e}$ and $C_{T}$, for the boundary CFT dual to the Einstein-Maxwell theory (3.1). This allows us to verify various expressions derived in section 2.3 for the expansion coefficients of the twist operator's conformal weight and magnetic response within the holographic framework of section 3.

To begin, we follow the calculation of [44] to evaluate the two-point correlator of a current dual to a bulk Maxwell field, however, we will be careful to include all of the numerical factors. This allows us to evaluate the constant $C_{V}$ appearing in eq. (2.52) for the Einstein-Maxwell theory studied in section 3. From [44], the bulk solution for the gauge field $A$, which near the AdS boundary approaches $\lim _{z \rightarrow 0} A \rightarrow \sum_{i} B_{i}(x) d x^{i}$, is given by

$$
A(z, x)=\mathcal{N} \int d^{d} x^{\prime}\left[\frac{z^{d-2}}{\left(z^{2}+\left(x-x^{\prime}\right)^{2}\right)^{d-1}} B_{i}\left(x^{\prime}\right) d x^{i}-z^{d-3} d z \frac{\left(x-x^{\prime}\right)^{i} B_{i}\left(x^{\prime}\right)}{\left(z^{2}+\left(x-x^{\prime}\right)^{2}\right)^{d-1}}\right],
$$

where the normalization constant is given by

$$
\mathcal{N}=\frac{\Gamma(d-1)}{\pi^{d / 2} \Gamma(d / 2-1)} .
$$

This coefficient $\mathcal{N}$ is chosen to ensure that

$$
\lim _{z \rightarrow 0} \mathcal{N} \frac{z^{d-2}}{\left(z^{2}+x^{2}\right)^{d-1}}=\delta^{d}(x) .
$$

Now our (Euclidean) Maxwell action (3.1) is given by

$$
I_{M a x}=\frac{\ell_{*}^{2}}{8 \ell_{\mathrm{P}}^{d-1}} \int d^{d} x d z \sqrt{G} G^{A C} G^{B D} F_{A B} F_{C D},
$$

where $A, B$ range over $i=1, \cdots d$ and $z$. Further, we work in Poincaré coordinates where $G_{A B}=\left(L^{2} / z^{2}\right) \delta_{A B}$. To extract the leading boundary contribution, we only need to consider the terms with $z$ derivatives. That is,

$$
I_{\text {Max }}=\frac{\ell_{*}^{2} L^{d-3}}{4 \ell_{\mathrm{P}}^{d-1}} \int \frac{d z d^{d} x}{z^{d-3}}\left[\left(\partial_{z} A_{i}\right)^{2}-2 \partial_{i} A_{z} \partial_{z} A_{i}+\left(\partial_{i} A_{z}\right)^{2}\right] .
$$

Using the bulk equations of motion for $A_{i}, A_{z}$ and integrating by parts, the above expression yields a boundary term at $z \rightarrow 0$ :

$$
\begin{aligned}
I_{\text {Max }} & =\lim _{z \rightarrow 0} \frac{\ell_{*}^{2} L^{d-3}}{4 \ell_{\mathrm{P}}^{d-1}} \int \frac{d^{d} x}{z^{d-3}}\left[A_{i} \partial_{z} A_{i}-A_{i} \partial_{i} A_{z}\right] \\
& =\frac{(d-1) \mathcal{N} \ell_{*}^{2} L^{d-3}}{4 \ell_{\mathrm{P}}^{d-1}} \iint d^{d} x_{1} d^{d} x_{2} \frac{B_{i}\left(x_{1}\right) B_{j}\left(x_{2}\right)}{\left|x_{12}\right|^{2 d-2}}\left(\delta_{i j}-2 \frac{x_{12}^{i} x_{12}^{j}}{\left|x_{12}\right|^{2}}\right),
\end{aligned}
$$


where the second line follows from substituting in eq. (C.9). Finally, we may differentiate this action twice with respect to the sources $B_{i}(x)$ to produce the two-point function of the corresponding current and we see the form matches precisely that given in eq. (2.52). Then we may read off the central charge $C_{V}$ in the boundary theory with the normalization of the bulk Maxwell term given in eq. (C.12):

$$
C_{V}=\frac{(d-1) \mathcal{N} \ell_{*}^{2} L^{d-3}}{2 \ell_{\mathrm{P}}^{d-1}}=\frac{\Gamma(d)}{2 \pi^{d / 2} \Gamma(d / 2-1)} \frac{\ell_{*}^{2} L^{d-3}}{\ell_{\mathrm{P}}^{d-1}} .
$$

Now eq. (2.53) relates this central charge to $(\hat{c}+\hat{e})$, the sum of the two CFT parameters which define the $\langle T J J\rangle$ correlator (2.37). Now for the boundary CFT dual to the Einstein-Maxwell theory (3.1), this correlator does not take the most general form possible $[45,46]$, i.e., $\hat{c}$ and $\hat{e}$ are not independent parameters. Rather one finds that

$$
\hat{c}=d(d-2) \hat{e} .
$$

Combining this constraint with eq. (2.53), we can also evaluate $\hat{c}$ and $\hat{e}$ for our holographic theory. In particular, we find

$$
\hat{e}=\frac{\Gamma\left(\frac{d+2}{2}\right)}{2 \pi^{d / 2}(d-1)^{2}} C_{V}=\frac{(d-2) \Gamma(d+1)}{16 \pi^{d}(d-1)^{2}} \frac{\ell_{*}^{2} L^{d-3}}{\ell_{\mathrm{P}}^{d-1}}
$$

and then $\hat{c}$ follows from eq. (C.16).

Finally, it is convenient to have the central charge $C_{T}$, which appears in the twopoint correlator of the stress tensor (2.27), for our holographic theory. In this case, the calculation analogous to that above for the Maxwell field was carried out for the metric in [47]. Hence we can simply quote the result for $C_{T}$ :

$$
C_{T}=\frac{\Gamma(d+2)}{\pi^{d / 2}(d-1) \Gamma(d / 2)} \frac{L^{d-1}}{\ell_{\mathrm{P}}^{d-1}} .
$$

\section{References}

[1] M. Levin and X.-G. Wen, "Detecting Topological Order in a Ground State Wave Function," Phys. Rev. Lett. 96 (2006) 110405 [arXiv:cond-mat/0510613];

A. Kitaev and J. Preskill, "Topological entanglement entropy," Phys. Rev. Lett. 96 (2006) 110404 [arXiv:hep-th/0510092];

A. Hamma, R. Ionicioiu and P. Zanardi, "Ground state entanglement and geometric entropy in the Kitaev's model," Phys. Lett. A 337 (2005) 22 [arXiv:quant-ph/0406202].

[2] P. Calabrese and J. L. Cardy, "Entanglement entropy and quantum field theory," J. Stat. Mech. 0406, P002 (2004) [arXiv:hep-th/0405152];

P. Calabrese and J. L. Cardy, "Entanglement entropy and quantum field theory: A non-technical introduction," Int. J. Quant. Inf. 4, 429 (2006) [arXiv:quant-ph/0505193]. 
[3] S. Ryu and T. Takayanagi, "Holographic derivation of entanglement entropy from AdS/CFT," Phys. Rev. Lett. 96 (2006) 181602 [arXiv:hep-th/0603001];

S. Ryu and T. Takayanagi, "Aspects of holographic entanglement entropy," JHEP 0608 (2006) 045 [arXiv:hep-th/0605073];

T. Nishioka, S. Ryu and T. Takayanagi, "Holographic Entanglement Entropy: An Overview," J. Phys. A 42 (2009) 504008 [arXiv:0905.0932 [hep-th]];

T. Takayanagi, "Entanglement Entropy from a Holographic Viewpoint," arXiv:1204.2450 [gr-qc].

[4] M. Van Raamsdonk, "Comments on quantum gravity and entanglement," arXiv:0907.2939 [hep-th];

M. Van Raamsdonk, "Building up spacetime with quantum entanglement," Gen. Rel. Grav. 42 (2010) 2323 [arXiv:1005.3035 [hep-th]].

[5] E. Bianchi and R. C. Myers, "On the Architecture of Spacetime Geometry," arXiv:1212.5183 [hep-th];

R. C. Myers, R. Pourhasan and M. Smolkin, "On Spacetime Entanglement," JHEP 1306 (2013) 013 [arXiv:1304.2030 [hep-th]];

V. Balasubramanian, B. Czech, B. D. Chowdhury and J. de Boer, "The entropy of a hole in spacetime," arXiv:1305.0856 [hep-th].

[6] A. Rényi, "On measures of information and entropy," in Proceedings of the 4th Berkeley Symposium on Mathematics, Statistics and Probability, 1, 547 (U. of California Press, Berkeley, CA, 1961);

A. Rényi, "On the foundations of information theory," Rev. Int. Stat. Inst. 33 (1965) 1.

[7] For example, see:

K. Zyczkowski, "Renyi extrapolation of Shannon entropy," Open Syst. Inf. Dyn. 10, 297 (2003) [arXiv:quant-ph/0305062];

C. Beck and F. Schlögl, "Thermodynamics of chaotic systems", (Cambridge University Press, Cambridge, 1993).

[8] H. Casini, M. Huerta and R. C. Myers, "Towards a derivation of holographic entanglement entropy," JHEP 1105 (2011) 036 [arXiv:1102.0440 [hep-th]].

[9] L.-Y. Hung, R. C. Myers, M. Smolkin and A. Yale, "Holographic Calculations of Renyi Entropy," JHEP 1112 (2011) 047 [arXiv:1110.1084 [hep-th]].

[10] I. R. Klebanov, S. S. Pufu, S. Sachdev and B. R. Safdi, "Renyi Entropies for Free Field Theories," JHEP 1204 (2012) 074 [arXiv:1111.6290 [hep-th]].

[11] A. Lewkowycz and J. Maldacena, "Generalized gravitational entropy," arXiv:1304.4926 [hep-th].

[12] G. Wong, I. Klich, L. A. P. Zayas and D. Vaman, "Entanglement Temperature and Entanglement Entropy of Excited States," arXiv:1305.3291 [hep-th]. 
[13] P. Caputa, G. Mandal and R. Sinha, "Dynamical entanglement entropy with angular momentum and U(1) charge," arXiv:1306.4974 [hep-th].

[14] T. Nishioka and I. Yaakov, "Supersymmetric Renyi Entropy," arXiv:1306.2958 [hep-th].

[15] H. Casini, "Entropy inequalities from reflection positivity," J. Stat. Mech. 1008 (2010) P08019 [arXiv:1004.4599 [quant-ph]];

B. Swingle, "Mutual information and the structure of entanglement in quantum field theory," arXiv:1010.4038 [quant-ph].

[16] L. Y. Hung, R. C. Myers and M. Smolkin, "Twist operators in higher dimensions," in preparation.

[17] M. Headrick, "Entanglement Renyi entropies in holographic theories," Phys. Rev. D 82 (2010) 126010 [arXiv:1006.0047 [hep-th]].

[18] A. Roberge and N. Weiss, "Gauge Theories With Imaginary Chemical Potential and the Phases of QCD," Nucl. Phys. B 275 (1986) 734.

[19] M. G. Alford, A. Kapustin and F. Wilczek, "Imaginary chemical potential and finite fermion density on the lattice," Phys. Rev. D 59 (1999) 054502 [hep-lat/9807039].

[20] E. Witten, "Constraints on Supersymmetry Breaking," Nucl. Phys. B 202 (1982) 253.

[21] R. C. Myers and A. Sinha, "Seeing a c-theorem with holography," Phys. Rev. D 82 (2010) 046006 [arXiv:1006.1263 [hep-th]];

R. C. Myers and A. Sinha, "Holographic c-theorems in arbitrary dimensions," JHEP 1101 (2011) 125 [arXiv:1011.5819 [hep-th]].

[22] E. Perlmutter, "A universal feature of CFT Renyi entropy," arXiv:1308.1083 [hep-th].

[23] H. Osborn and A. C. Petkou, "Implications of conformal invariance in field theories for general dimensions," Annals Phys. 231 (1994) 311 [hep-th/9307010].

[24] T. Takayanagi, unpublished.

[25] H. Casini and M. Huerta, "Entanglement entropy in free quantum field theory," J. Phys. A 42 (2009) 504007 [arXiv:0905.2562 [hep-th]];

H. Casini, C. D. Fosco and M. Huerta, "Entanglement and alpha entropies for a massive Dirac field in two dimensions," J.Stat.Mech. 0507 (2005) P07007 [arXiv:cond-mat/0505563];

T. Azeyanagi, T. Nishioka and T. Takayanagi, "Near Extremal Black Hole Entropy as Entanglement Entropy via AdS(2)/CFT(1)," Phys. Rev. D 77 (2008) 064005 [arXiv:0710.2956 [hep-th]].

[26] A. Belin, A. Maloney and S. Matsuura, "Holographic Phases of Renyi Entropies," arXiv:1306.2640 [hep-th]. 
[27] R.-G. Cai and A. Wang, "Thermodynamics and stability of hyperbolic charged black holes," Phys. Rev. D 70 (2004) 064013 [hep-th/0406057].

[28] S. A. Hartnoll, C. P. Herzog and G. T. Horowitz, "Holographic Superconductors," JHEP 0812 (2008) 015 [arXiv:0810.1563 [hep-th]].

[29] A. Belin, L. Y. Hung, A. Maloney and S. Matsuura, JHEP 1501, 059 (2015) [arXiv:1407.5630 [hep-th]].

[30] M. Cvetic, S. Nojiri and S. D. Odintsov, "Black hole thermodynamics and negative entropy in de Sitter and anti-de Sitter Einstein-Gauss-Bonnet gravity," Nucl. Phys. B 628 (2002) 295 [hep-th/0112045].

[31] X.-H. Ge, Y. Matsuo, F.-W. Shu, S.-J. Sin and T. Tsukioka, "Viscosity Bound, Causality Violation and Instability with Stringy Correction and Charge," JHEP 0810 (2008) 009 [arXiv:0808.2354 [hep-th]].

[32] D. Anninos and G. Pastras, "Thermodynamics of the Maxwell-Gauss-Bonnet anti-de Sitter Black Hole with Higher Derivative Gauge Corrections," JHEP 0907 (2009) 030 [arXiv:0807.3478 [hep-th]].

[33] A. Belin, L.-Y. Hung, A. Maloney, S. Matsuura, R.C. Myers and T. Sierens, in preparation.

[34] A. Grigor'yan and M. Noguchi, "The heat kernel on hyperbolic space, " Bull. London Math. Soc. 30 (1998) 643;

A. Grigor'yan, "Upper bounds on a complete non compact manifold, " J. Funct. Anal. 127 (1995) 363;

A. Debiard, B. Gaveau, E. Mazet, " Theoreme de comparison in geometrie riemannienne," Publ. Res. Inst. Math. Sci. Kyoto 12 (1976) 391;

R. Camporesi and A. Higuchi, "Spectral functions and zeta functions in hyperbolic spaces," J. Math. Phys. 35 (1994) 4217;

R. Camporesi, Commun. Math. Phys. 148 (1992) 283;

A. Lewkowycz, R. C. Myers and M. Smolkin, "Observations on entanglement entropy in massive QFT's," JHEP 1304 (2013) 017 [arXiv:1210.6858 [hep-th]].

[35] R. Camporesi, "Harmonic analysis and propagators on homogeneous spaces," Phys.

Rept. 196 (1990) 1;

R. Camporesi, "The Spinor heat kernel in maximally symmetric spaces," Commun. Math. Phys. 148 (1992) 283;

A. A. Bytsenko, G. Cognola, L. Vanzo and S. Zerbini, "Quantum fields and extended objects in space-times with constant curvature spatial section," Phys. Rept. 266 (1996) 1 [hep-th/9505061].

[36] C. Martinez, C. Teitelboim and J. Zanelli, "Charged rotating black hole in three space-time dimensions," Phys. Rev. D 61 (2000) 104013 [hep-th/9912259]. 
[37] I. R. Klebanov and E. Witten, "AdS/CFT correspondence and symmetry breaking," Nucl. Phys. B 556 (1999) 89 [hep-th/9905104].

[38] L.-Y. Hung and A. Sinha, "Holographic quantum liquids in 1+1 dimensions," JHEP 1001 (2010) 114 [arXiv:0909.3526 [hep-th]].

[39] A. Ejaz, H. Gohar, H. Lin, K. Saifullah and S.-T. Yau, "Quantum tunneling from three-dimensional black holes," arXiv:1306.6380 [hep-th].

[40] P. Kraus, "Lectures on black holes and the $\operatorname{AdS}(3) / \mathrm{CFT}(2)$ correspondence," Lect. Notes Phys. 755 (2008) 193 [hep-th/0609074].

[41] V. Balasubramanian and P. Kraus, "A Stress tensor for Anti-de Sitter gravity," Commun. Math. Phys. 208 (1999) 413 [hep-th/9902121];

R. Emparan, C. V. Johnson and R. C. Myers, "Surface terms as counterterms in the AdS/CFT correspondence," Phys. Rev. D 60 (1999) 104001 [hep-th/9903238].

[42] J. D. Brown and J. W. York, Jr., "Quasilocal energy and conserved charges derived from the gravitational action," Phys. Rev. D 47, 1407 (1993) [gr-qc/9209012].

[43] R. C. Myers, "Stress tensors and Casimir energies in the AdS / CFT correspondence," Phys. Rev. D 60 (1999) 046002 [hep-th/9903203].

[44] E. Witten, "Anti-de Sitter space and holography," Adv. Theor. Math. Phys. 2 (1998) 253 [hep-th/9802150].

[45] D. M. Hofman and J. Maldacena, "Conformal collider physics: Energy and charge correlations," JHEP 0805 (2008) 012 [arXiv:0803.1467 [hep-th]].

[46] D. Chowdhury, S. Raju, S. Sachdev, A. Singh and P. Strack, "Multipoint correlators of conformal field theories: implications for quantum critical transport," Phys. Rev. B 87 (2013) 085138 [arXiv:1210.5247 [cond-mat.str-el]].

[47] A. Buchel, J. Escobedo, R. C. Myers, M. F. Paulos, A. Sinha and M. Smolkin, "Holographic GB gravity in arbitrary dimensions," JHEP 1003 (2010) 111 [arXiv:0911.4257 [hep-th]]. 